\title{
Chemical composition and therapeutic mechanism of Xuanbai Chengqi Decoction in the treatment of COVID-19 by network pharmacology, molecular docking and molecular dynamic analysis
}

\author{
Liming Fan ${ }^{1} \cdot$ Shuai Feng ${ }^{1} \cdot$ Ting Wang ${ }^{1} \cdot$ Xinli Ding $^{1} \cdot$ Xinxin An $^{1} \cdot$ Zhen Wang $^{1} \cdot$ Kun Zhou $^{1} \cdot$ Minjuan Wang $^{2}$. \\ Xifeng Zhai ${ }^{3}$. Yang $\mathrm{Li}^{1}$
}

Received: 4 January 2022 / Accepted: 18 February 2022 / Published online: 8 March 2022

(c) The Author(s), under exclusive licence to Springer Nature Switzerland AG 2022

\begin{abstract}
Xuanbai Chengqi Decoction (XBCQD), a classic traditional Chinese medicine, has been widely used to treat COVID-19 in China with remarkable curative effect. However, the chemical composition and potential therapeutic mechanism is still unknown. Here, we used multiple open-source databases and literature mining to select compounds and potential targets for XBCQD. The COVID-19 related targets were collected from GeneCards and NCBI gene databases. After identifying putative targets of XBCQD for the treatment of COVID-19, PPI network was constructed by STRING database. The hub targets were extracted by Cytoscape 3.7.2 and MCODE analysis was carried out to extract modules in the PPI network. R 3.6.3 was used for GO enrichment and KEGG pathway analysis. The effective compounds were obtained via network pharmacology and bioinformatics analysis. Drug-likeness analysis and ADMET assessments were performed to select core compounds. Moreover, interactions between core compounds and hub targets were investigated through molecular docking, molecular dynamic (MD) simulations and MM-PBSA calculations. As a result, we collected 638 targets from 61 compounds of XBCQD and 845 COVID-19 related targets, of which 79 were putative targets. Based on the bioinformatics analysis, 10 core compounds and 34 hub targets of XBCQD for the treatment of COVID-19 were successfully screened. The enrichment analysis of GO and KEGG indicated that XBCQD mainly exerted therapeutic effects on COVID-19 by regulating signal pathways related to viral infection and inflammatory response. Meanwhile, the results of molecular docking showed that there was a stable binding between the core compounds and hub targets. Moreover, MD simulations and MM-PBSA analyses revealed that these compounds exhibited stable conformations and interacted well with hub targets during the simulations. In conclusion, our research comprehensively explained the multi-component, multi-target, and multi-pathway intervention mechanism of XBCQD in the treatment of COVID-19, which provided evidence and new insights for further research.
\end{abstract}

Yang Li

ly2011@nwu.edu.cn

1 Biomedicine Key Laboratory of Shaanxi Province, College of Life Sciences, Northwest University, Xi' an 710069, China

2 Physical and Chemical Laboratory, Shaanxi Provincial Center for Disease Control and Prevention, Xi' an 710054, China

3 School of Pharmaceutical Sciences, Xi' an Medical University, Xi' an 710021, China 


\section{Graphical abstract}

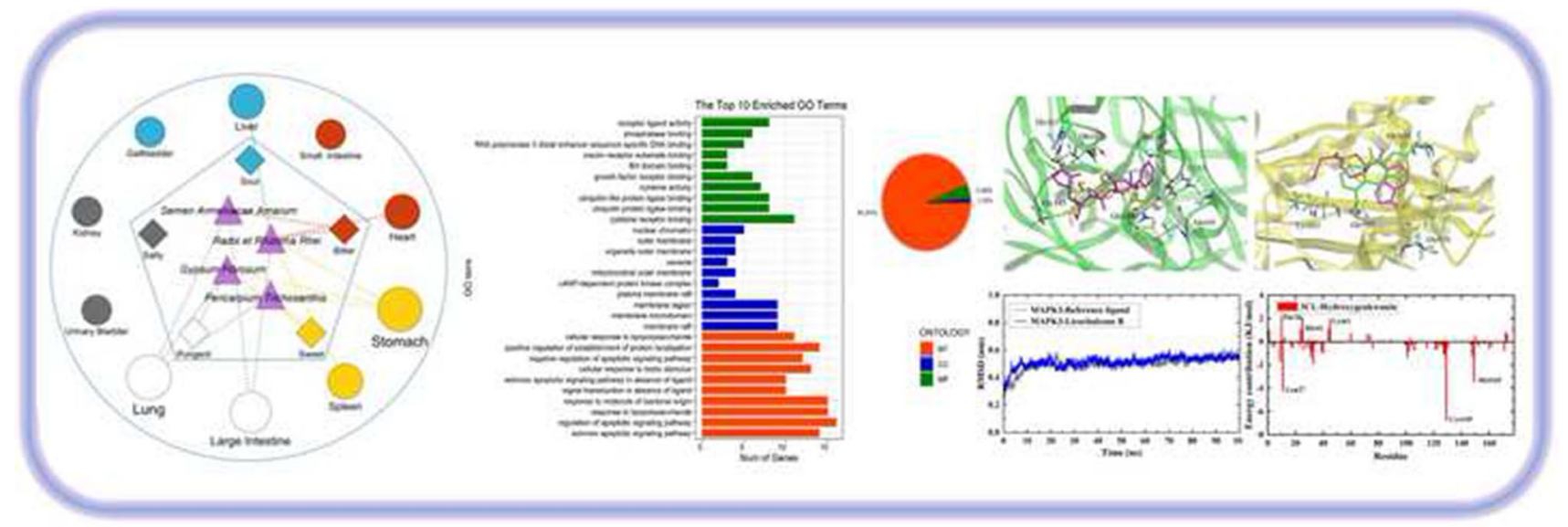

Keywords COVID-19 $\cdot$ Traditional Chinese medicine $\cdot$ Xuanbai Chengqi Decoction $\cdot$ Network pharmacology $\cdot$ Molecular docking $\cdot$ Molecular dynamic

\section{Introduction}

The Coronavirus Disease 2019 (COVID-19) is caused by Severe Acute Respiratory Syndrome Coronavirus 2 (SARSCoV-2), which broke out in December 2019 and spread rapidly around the world [1]. SARS-CoV-2 belongs to the Coronaviridae family in the order Nidovirales and the subfamily of Orthocoronavirinae, including 4 structural proteins: spike $(\mathrm{S})$ protein, membrane $(\mathrm{M})$ protein, nucleocapsid $(\mathrm{N})$ protein and envelope (E) protein [2]. The virus may enter epithelial cells through SARS-CoV-2 S protein binding to Angiotensin Converting Enzyme 2 (ACE2) receptor. The combination of virus and receptor leads to the fusion of the viral envelope and cell membrane with entry of the viral nucleocapsid into the cell, and the viral RNA hijack the host cell ribosome to translate the virus code, resulting in the production of viral proteins and viral replication [3]. The common clinical symptoms of COVID-19 are cough, fever, fatigue or myalgia, and possible complications include septicemia, respiratory distress syndrome, or multiple organ failure [4]. According to COVID-19 Dashboard by the Center for Systems Science and Engineering (CSSE) of Johns Hopkins University (JHU), as of January 4, 2022, a total of 292,567,808 people worldwide was infected with the SARS-CoV-2, of whom 5,449,575 died. The COVID-19 epidemic has seriously affected people's health, economic development, and social progress all over the world [5]. Although some COVID-19 vaccines have been approved, they are not available in all countries infected with SARS-CoV-2 [6]. With the persevering efforts of researchers, several options have been envisaged to control the emerging infections of COVID-19, such as monoclonal antibodies, peptides, oligonucleotide-based therapies, interferon therapies and small molecule drugs. However, these new interventions may take months to years to develop [7]. Therefore, the fast and effective drugs to combat the COVID-19 epidemic are urgently needed.

Traditional Chinese Medicine (TCM) has been in clinical practice for more than two thousand years and has a unique superiority in the prevention and treatment of infectious diseases. TCM has shown certain effects in the treatment of Severe Acute Respiratory Syndrome (SARS) and Middle East Respiratory Syndrome (MERS) [8]. And now, TCM also plays an important role in COVID-19. In the trial 8th edition of the "Guideline on Diagnosis and Treatment of COVID-19" issued by the National Health Commission of China on August 18, 2020, some TCM prescriptions were recorded as recommended prescription for COVID-19. Xuanbai Chengqi Decoction (XBCQD) adapted from the "Identification of Warm Disease" written by Wu Ju-tong in the Qing Dynasty, which is a classic clinical prescription for the treatment of lung diseases $[9,10]$. The formula consists of 4 Chinese medicines including Radix et Rhizoma Rhei (Dahuang), Pericarpium Trichosanthis (Gualoupi), Semen Armeniacae Amarum (Kuxingren) and Gypsum Fibrosum (Shigao). Radix et Rhizoma Rhei has the effect of discharging damp-heat of large intestine, which corresponds to the diarrhea syndrome in some patients [11]. Pericarpium Trichosanthis can reduce phlegm and dissipate blood stagnation [12]. Semen Armeniacae Amarum is effective in treating of cough and asthma accompanied by stuffiness in the chest and profuse expectoration. Gypsum Fibrosum is mainly used for febrile diseases due to exogenous affection with high fever, dire thirst, asthma, and cough caused by heat in the lung [13]. Modern pharmacological researches have 
demonstrated that XBCQD can significantly alleviate the pulmonary inflammatory responses in patients with acute respiratory distress syndrome, improve static/dynamic lung compliance and reduce the incidence of complications [9]. It was found that XBCQD has a protective effect on cecal ligation and puncture-induced septic lung injury, and could reduce the level of TNF- $\alpha$, IL- $1 \beta$ and IL-10 in bronchoalveolar lavage fluids [14]. Furthermore, XBCQD has shown significant efficacy in the treatment of COVID-19 and has been widely used in clinical practice $[15,16]$. However, due to the complex components of TCM prescription, it is difficult to elucidate the chemical composition and potential mechanism of XBCQD against COVID-19 through traditional pharmacological methods.

Network pharmacology is a new biological analysis technology in recent years, which is considered to have the potential to discover drugs by combining with systems biology, bioinformatics, and polypharmacology [17]. This technology is consistent with the theory of TCM and can reveal the therapeutic mechanism of drugs by establishing a complex network of drugs, targets, pathways, and diseases. Therefore, many researchers have used network pharmacology to explore the material basis of Chinese medicine and predict the mechanism of TCM prescription [8]. Molecular docking is a virtual screening technology used to explore the behavior of molecule at the binding site of a target protein by simulating the geometric structure and interaction between molecule and protein [18]. Molecular dynamic (MD) simulation is an efficient computer simulation method for investigating biological phenomena and molecular mechanisms of interactions between biological macromolecules and ligands [19].

The purpose of this research is to systematically explore the chemical composition and therapeutic mechanism of XBCQD on COVID-19. After extensive data mining, we have obtained effective compounds and related targets. Then, integrate network pharmacology, molecular docking, and MD methods to analyze the interactions between the compounds of XBCQD, the targets and COVID-19. The flowchart of the research is shown in Fig. 1.

\section{Materials and methods}

\section{Collection of XBCQD meridian tropisms, compounds, and targets}

The TCM meridian tropism information of the 4 Chinese medicines in XBCQD was obtained from the 2020 edition of the Chinese Pharmacopoeia. Except for Gypsum Fibrosum, all the compounds in XBCQD were derived from the Traditional Chinese Medicine Systems Pharmacology Database and Analysis Platform (TCMSP) (http://tcmspw.com/ tcmsp.php) and Bioinformatics Analysis Tool for Molecular Mechanism of Traditional Chinese Medicine (BATMANTCM) (http://bionet.ncpsb.org.cn/batman-tcm/), and were screened by the metrics of oral bioavailability $(\mathrm{OB}) \geq 30 \%$ and drug-likeness (DL) $\geq 0.18$ [20-23]. The compounds of Gypsum Fibrosum were collected from the Traditional Chinese Medicines Integrated Database (TCMID) (http://www. megabionet.org/tcmid/) [24]. In order to make the results more credible, we supplemented the bioactive compounds that did not meet the screening criteria through literature mining. Subsequently, TCMSP, the Encyclopedia of Traditional Chinese Medicine (ETCM) (http://www.tcmip.cn/ ETCM/) and SwissTargetPrediction (http://swisstargetpred iction.ch/) were employed to identify potential targets of the compounds, except calcium sulfate dihydrate, which used PharmMapper (http://lilab-ecust.cn/pharmmapper/index. html) and TargetNet (http://targetnet.scbdd.com/home/ index/) databases [25-28]. The screening criteria for protein targets of XBCQD compounds were set as follows: In ETCM database, the targets were selected with the prediction confidence index more than 0.8. In PharmMapper database, we set Zscore greater than 0 to filter targets. In SwissTargetPrediction and TargetNet databases, the targets with probability more than 0 were selected as potential targets of the compounds [29].

\section{Screening of COVID-19 related targets and putative targets of XBCQD}

COVID-19 related targets were acquired from GeneCards (https://www.genecards.org/) and NCBI (https://www.ncbi. nlm.nih.gov/), with "COVID-19", "novel coronavirus" and "severe acute respiratory syndrome coronavirus 2" as keywords [30]. To ensure the comprehensiveness and accuracy of target screening, we set search parameters in the database as follows: In the GeneCards database, the targets with relevance scores $>0$ were selected. In the NCBI database, we selected species as "Homo sapiens" to screen the targets. All the above targets were normalized into official gene symbols via UniProt database (http://www.uniprot.org/) [31]. Subsequently, the VennDiagram program package in the R 3.6.3 software was used to map disease targets and compound targets, and define their intersection targets as putative targets of XBCQD in the treatment of COVID-19.

\section{Protein-protein interaction (PPI) network and molecular complex detection (MCODE) analysis}

The PPI network was constructed through STRING database (https://string-db.org/) to explore interaction between the putative targets of XBCQD in COVID-19 treatment, with the species limited to "Homo sapiens" [32]. We selected a confidence score threshold greater than 0.9 in 
Fig. 1 The workflow of exploring the therapeutic mechanism of XBCQD on COVID-19
Database

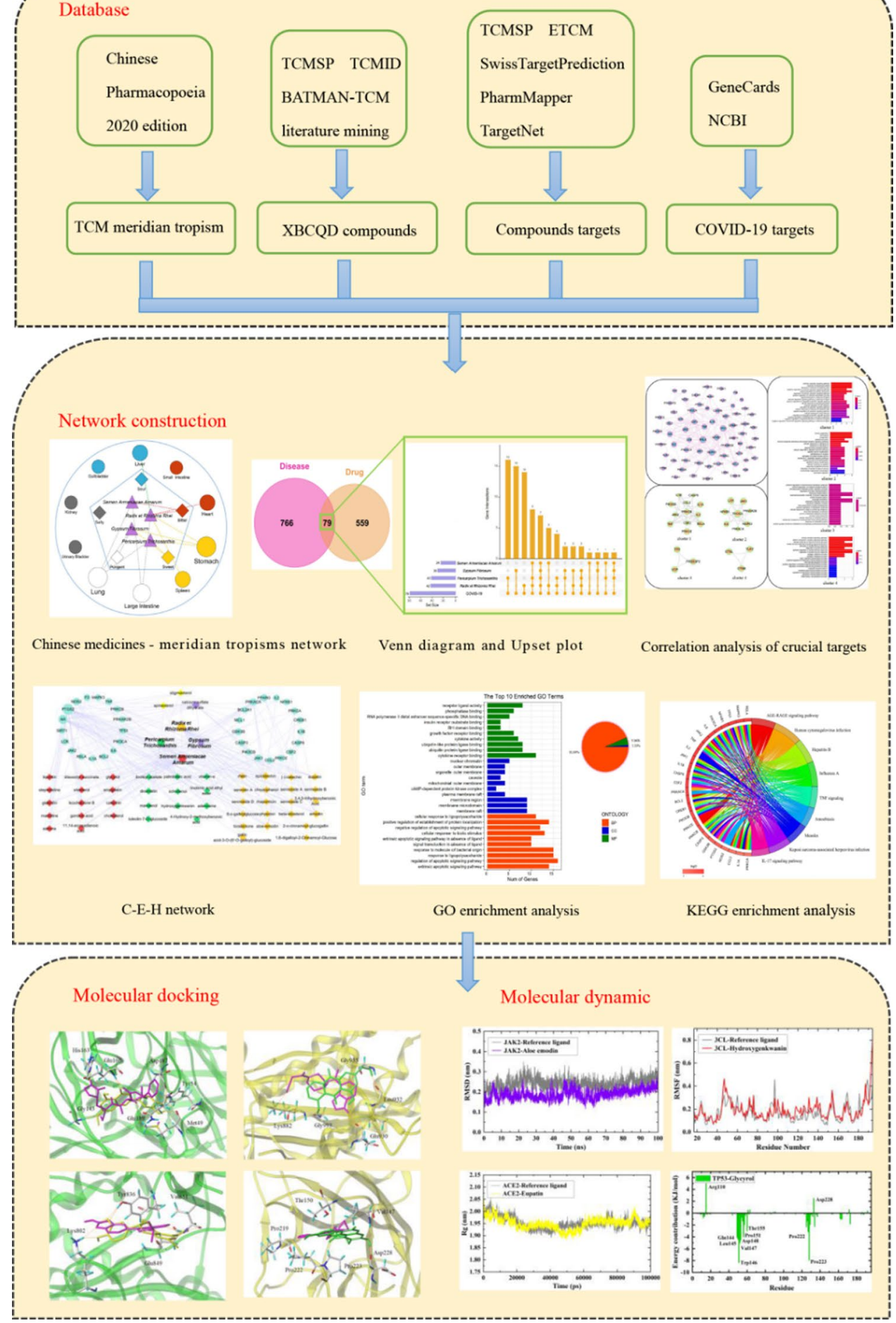

STRING platform to construct the PPI network to meet the accuracy and identify crucial targets. The visualization and topology analysis of PPI network were achieved by Cytoscape 3.7.2. Then, MCODE analysis was carried out to extract densely connected modules in the PPI network. The MCODE algorithm is based on vertex weighting by local neighborhood density and outward traversal from a locally dense seed protein to separate the dense regions by certain connectivity parameters, which can fine-tune the clusters of interest and monitoring the connectivity of the cluster. To further explore the functional connections of the targets in the clusters, biological process analysis was predicted by R 3.6.3 software. 


\section{Construction of the Chinese medicines-effective compounds-hub targets (C-E-H) network of XBCQD and enrichment analysis}

In the PPI network, the degree value is an important indicator to evaluate the role of the target in the network. Based on the above PPI data, the crucial target that was greater than or equal to the median degree was defined as hub target, which was inputted to the Cytoscape 3.7.2 to construct the C-E-H network of XBCQD. The compounds linking the hub targets were considered to be effective compounds in XBCQD. Next, the topology features of the C-E-H network were analyzed by NetworkAnalyzer plugin of Cytoscape 3.7.2. Meanwhile, we defined the effective compounds with degree greater than the median degree as the core compounds for further analysis. In order to explore the mechanism of XBCQD in the treatment of COVID-19, R 3.6.3 software was used to conduct Gene Ontology (GO) and Kyoto Encyclopedia of Genes and Genomes (KEGG) pathway analysis. Afterwards, the bar plot and chord plot were drawn with the ggplot2 and GOplot packages in R 3.6.3 software.

\section{Drug-likeness analysis and ADMET evaluation}

The structures of the core compounds were screened to predict whether these compounds obey Lipinski's rule of five. At the same time, the rules of Pfizer and Golden triangle were also used to verify drug-like properties [33]. The physicochemical and pharmacokinetic properties of core compounds were evaluated using ADMETlab 2.0 (https:// admetmesh.scbdd.com/), which systematically assesses the absorption, distribution, metabolism, excretion, and toxicity (ADMET) of compounds based on a comprehensively collected database $[34,35]$.

\section{Molecular docking analysis}

Molecular docking is a key technology to verify the interaction between small molecule ligands and protein receptors. The 3D structures of the selected core compounds of XBCQD were searched through PubChem database (https:// pubchem.ncbi.nlm.nih.gov/) [36]. The sdf files were then converted to mol2 files using Sybyl-X 2.1.1 software and minimized under Tripos force field with Gasteiger-Huckel charge. The crystal structures of hub targets were downloaded from RCSB Protein Data Bank (https://www.rcsb. org/) and were imported into Surflex-Dock module of Sybyl-X 2.1.1 [37]. The proteins were prepared by removing ligands and crystallographic water molecules, adding hydrogens, and filling in missing side chains. Co-crystallized ligands or inhibitors were used to define the active binding pocket and generated the protomols. Residues with a radius of $5.0 \AA$ around the ligand were chosen as the active sites
[38]. The other docking parameters were kept at default. Total score of Surflex-Dock was calculated to estimate the ligand-receptor interactions, with binding affinity expressed in $-\log _{10}(\mathrm{Kd})$ units [39]. In general, Total Score $>4.5$ suggests a certain binding activity, Total Score $>5.0$ suggests a good binding activity, and Total Score $>7.0$ suggests a strong binding activity [40].

\section{Molecular dynamic (MD) simulation}

The MD simulation was carried out to verify the structural and conformational stability of the ligand-receptor complexes in molecular docking. The protein macromolecule was subjected to MD using a GROMOS96 54a7 force field in GROMACS 2019.6, and the initial topology of the selected core compound was generated using ATB online server (http://atb.uq.edu.au/) [41, 42]. The system was solvated using simple point charges (SPC) water model in a dodecahedron box, and $\mathrm{Na}^{+}$and $\mathrm{Cl}^{-}$ions were added to maintain the overall neutrality of the system. The complex system energy was minimized using 50,000 steps steepest descent algorithm. After that, the system was equilibrated for 100 ps using NVT canonical ensemble followed by NPT isothermal-isobaric ensemble, respectively. The particlemesh Ewald (PME) method was used for long-range electrostatic interactions [43]. Finally, we performed $100 \mathrm{~ns}$ of MD simulation on representative complexes. Molecular dynamics trajectories were analyzed using gmx_rms, gmx_rmsf, gmx_gyrate and gmx_hbond tools in the GROMACS suite, with analysis parameters including root mean square deviation (RMSD), root mean square fluctuation (RMSF), radius of gyration ( $\mathrm{Rg}$ ) and hydrogen bonds interactions (H-bonds). MM-PBSA calculations of the complexes were carried out using g_mmpbsa tool, which calculated the binding free energies of the complexes and the individual energy contributions of the residues [44]. Xmgrace (Grace 5.1.21) was used to plot the graphs [45].

\section{Results}

\section{Meridian tropism information of Chinese medicines in XBCQD}

According to the medicinal properties and meridian tropisms theory of TCM, the 4 Chinese medicines of XBCQD were classified. The Chinese medicines-meridian tropisms network based on meridian tropism information was built by Cytoscape 3.7.2 (Fig. 2). The two nodes with the highest degree of connection in the network were the lung and stomach, followed by the large intestine, spleen, liver, heart, kidney, gallbladder, small intestine and urinary bladder. Three Chinese medicines in XBCQD were associated with 


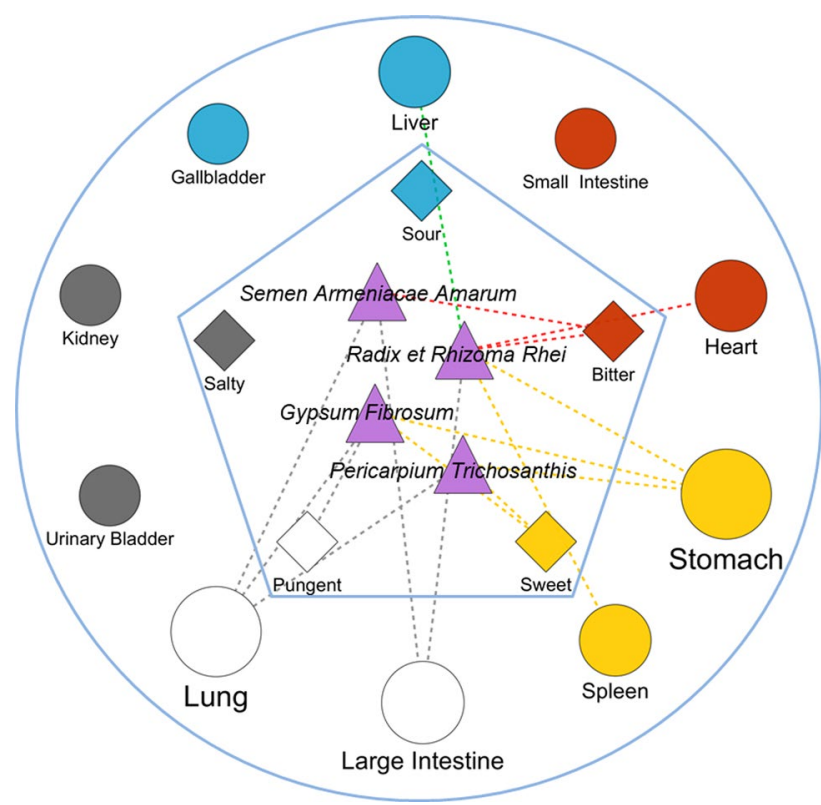

Fig. 2 The Chinese medicines-meridian tropisms network of XBCQD. The network shows the links of the Chinese medicines in $\mathrm{XBCQD}$ and their corresponding information about flavors(sour, bitter, sweet, pungent and salty) and their meridian tropisms(Liver, Heart, Spleen, Lung, Kidney, Gallbladder, Small Intestine, Stomach, Large Intestine, and Urinary Bladder). The triangle, diamond and circular represent the Chinese medicines, flavors and meridians, respectively

the Lung meridian, namely Semen Armeniacae Amarum, Pericarpium Trichosanthis and Gypsum Fibrosum. Among the flavor nodes, the two with the greatest degree of connection were sweet and bitter. The results reflected the treatment concept of holistic therapy of TCM. Meanwhile, it also suggested XBCQD may have specifically role in the treatment of lung and stomach diseases.

\section{Collection of compounds and targets in XBCQD}

A total of 61 compounds of XBCQD were selected from TCMSP, BATMAN-TCM, TCMID databases and literature mining after screening and deduplication. Among them, 27 compounds were obtained from Radix et Rhizoma Rhei, and 15 of them were collected from literature mining, involving emodin, chrysophanol, physcion, sennoside A, sennoside $\mathrm{B}$, sennoside $\mathrm{C}$, sennoside $\mathrm{D}$ and sennidin $\mathrm{A}$, etc. [46-49]. There were 18 compounds in Semen Armeniacae Amarum and 1 compound was collected by literature [50]. Pericarpium Trichosanthis contained 17 compounds, 6 of which came from literature mining, including 4-hydroxy2-methoxybenzoic acid, luteolin-7-O-glucoside, palmitoleic acid, glycerol monopalmitate, oxofangchirine and adenosine $[51,52]$. Only 1 compound was included in Gypsum Fibrosum, namely calcium sulfate dihydrate. Next, a total of 638 potential targets corresponding to the above compounds were acquired from TCMSP, ETCM, SwissTargetPrediction, PharmMapper and TargetNet databases after screening and removing the duplicates, among which the numbers of targets of Radix et Rhizoma Rhei, Pericarpium Trichosanthis, Semen Armeniacae Amarum and Gypsum Fibrosum were 214, 339, 265 and 209, respectively. The information of compounds in XBCQD was shown in Table 1.

\section{Collection of COVID-19 related targets and identification of putative targets}

Searched for targets related to COVID-19 from GeneCards and NCBI genes. After removing repetitive targets, a total of 845 targets were collected as disease targets. Further, the 638 targets corresponding to the compounds of XBCQD were mapped to 845 COVID-19 related targets. Finally, 79 putative targets for XBCQD to treat COVID-19 were identified (Fig. 3a, Table 2). As shown in Fig. 3b and Table S1, the Upset plot reflected the distribution of 79 putative targets in 4 Chinese medicines. The number of putative targets in Radix et Rhizoma Rhei, Pericarpium Trichosanthis, Gypsum Fibrosum and Semen Armeniacae Amarum was 42, 41, 30 and 24, respectively. Furthermore, COVID-19 and 4 Chinese medicines of XBCQD had some common targets. For instance, there were 7 common targets in Radix et Rhizoma Rhei, Pericarpium Trichosanthis, Gypsum Fibrosum, Semen Armeniacae Amarum and COVID-19, including NOS2, AR, JAK1, PTGS1, SIGMAR1, DNMT1 and PTGS2. The distribution of common targets indicated the synergistic effect of 4 Chinese medicines of XBCQD in the treatment of COVID-19.

\section{Construction of protein-protein interaction (PPI) network and analysis of clusters}

The PPI analysis was performed on 79 putative targets through STRING database. After deleting the disconnected nodes and setting the minimum confidence score of the network to 0.9 , we exported the result as a tsv file and imported it into the Cytoscape 3.7.2 software for target relationship mining and topology analysis. As shown in Fig. 4a, there were 67 crucial nodes and 195 edges, with a median degree value of 5 . The size of the nodes are proportional to the degree. The larger the node, the greater the degree value. Finally, 34 hub targets were identified in the PPI network, including RELA, MAPK3, TP53, NFkB1, PIK3CA, IL6, JAK2, TNF, IL2, JAK1, IL1B, CASP8, CSF2, LCK, PRKACA, BCL2, CREB1, SIRT1, PIK3CB, PRKCE, PRKCB, PPARG, CASP3, GSK3B, AR, PRKAR2B, F2, PTGS2, MCL1, BCL2A1, NOS2, CCL2, IL1A and PRKCA. These hub targets played a pivotal role in the treatment of COVID-19. 
Table 1 The information of the compounds in XBCQD

\begin{tabular}{|c|c|c|c|c|c|}
\hline Molecular Name & Molecular Formula & Molecular Weight & OB $(\%)$ & DL & Chinese medicine \\
\hline Eupatin & $\mathrm{C}_{18} \mathrm{H}_{16} \mathrm{O}_{8}$ & 360.30 & 50.80 & 0.41 & Radix et Rhizoma Rhei \\
\hline Physciondiglucoside & $\mathrm{C}_{28} \mathrm{H}_{32} \mathrm{O}_{15}$ & 608.50 & 41.65 & 0.63 & Radix et Rhizoma Rhei \\
\hline Rhein & $\mathrm{C}_{15} \mathrm{H}_{8} \mathrm{O}_{6}$ & 284.22 & 47.07 & 0.28 & Radix et Rhizoma Rhei \\
\hline Torachrysone-8-glucoside & $\mathrm{C}_{20} \mathrm{H}_{24} \mathrm{O}_{9}$ & 408.40 & 43.02 & 0.74 & Radix et Rhizoma Rhei \\
\hline Toralactone & $\mathrm{C}_{15} \mathrm{H}_{12} \mathrm{O}_{5}$ & 272.25 & 46.46 & 0.24 & Radix et Rhizoma Rhei \\
\hline Emodin-1-O-beta-D-glucopyranoside & $\mathrm{C}_{21} \mathrm{H}_{20} \mathrm{O}_{10}$ & 432.40 & 44.81 & 0.80 & Radix et Rhizoma Rhei \\
\hline Daucosterol & $\mathrm{C}_{35} \mathrm{H}_{60} \mathrm{O}_{6}$ & 576.80 & 37.23 & 0.76 & Radix et Rhizoma Rhei \\
\hline Beta-sitosterol & $\mathrm{C}_{29} \mathrm{H}_{50} \mathrm{O}$ & 414.70 & 36.91 & 0.75 & Radix et Rhizoma Rhei \\
\hline Aloe-emodin & $\mathrm{C}_{15} \mathrm{H}_{10} \mathrm{O}_{5}$ & 270.24 & 83.38 & 0.24 & Radix et Rhizoma Rhei \\
\hline Gallic acid-3-O-(6'-O-galloyl)-glucoside & $\mathrm{C}_{20} \mathrm{H}_{20} \mathrm{O}_{14}$ & 484.36 & 30.25 & 0.67 & Radix et Rhizoma Rhei \\
\hline (-)-Catechin & $\mathrm{C}_{15} \mathrm{H}_{14} \mathrm{O}_{6}$ & 290.27 & 49.68 & 0.24 & Radix et Rhizoma Rhei \\
\hline Epicatechin & $\mathrm{C}_{15} \mathrm{H}_{14} \mathrm{O}_{6}$ & 290.27 & 48.96 & 0.24 & Radix et Rhizoma Rhei \\
\hline Emodin* & $\mathrm{C}_{15} \mathrm{H}_{10} \mathrm{O}_{5}$ & 270.24 & 24.40 & 0.24 & Radix et Rhizoma Rhei \\
\hline Chrysophanol* & $\mathrm{C}_{15} \mathrm{H}_{10} \mathrm{O}_{4}$ & 254.24 & 18.64 & 0.21 & Radix et Rhizoma Rhei \\
\hline Physcion* & $\mathrm{C}_{16} \mathrm{H}_{12} \mathrm{O}_{5}$ & 284.26 & 22.29 & 0.27 & Radix et Rhizoma Rhei \\
\hline Sennoside B* & $\mathrm{C}_{42} \mathrm{H}_{38} \mathrm{O}_{20}$ & 862.70 & 3.34 & 0.08 & Radix et Rhizoma Rhei \\
\hline Sennoside $A^{*}$ & $\mathrm{C}_{42} \mathrm{H}_{38} \mathrm{O}_{20}$ & 862.70 & 3.34 & 0.08 & Radix et Rhizoma Rhei \\
\hline Sennoside $C^{*}$ & $\mathrm{C}_{42} \mathrm{H}_{40} \mathrm{O}_{19}$ & 848.80 & 3.99 & 0.09 & Radix et Rhizoma Rhei \\
\hline Sennoside D* & $\mathrm{C}_{42} \mathrm{H}_{40} \mathrm{O}_{19}$ & 848.80 & 3.99 & 0.09 & Radix et Rhizoma Rhei \\
\hline Sennidin A* & $\mathrm{C}_{30} \mathrm{H}_{18} \mathrm{O}_{10}$ & 538.5 & 74.55 & 0.57 & Radix et Rhizoma Rhei \\
\hline 1,6-Digalloyl-2-Cinnamoylglucose* & $\mathrm{C}_{29} \mathrm{H}_{26} \mathrm{O}_{15}$ & 614.52 & 17.02 & 0.22 & Radix et Rhizoma Rhei \\
\hline Rhaponticin* & $\mathrm{C}_{21} \mathrm{H}_{24} \mathrm{O}_{9}$ & 420.40 & 9.00 & 0.55 & Radix et Rhizoma Rhei \\
\hline Rhapontigenin* & $\mathrm{C}_{15} \mathrm{H}_{14} \mathrm{O}_{4}$ & 258.27 & 76.25 & 0.15 & Radix et Rhizoma Rhei \\
\hline 6-O-galloyl-glucose* & $\mathrm{C}_{13} \mathrm{H}_{16} \mathrm{O}_{10}$ & 332.26 & 17.19 & 0.23 & Radix et Rhizoma Rhei \\
\hline 2-O-cinnamoyl-glucogallin* & $\mathrm{C}_{22} \mathrm{H}_{22} \mathrm{O}_{11}$ & 462.40 & 30.00 & 0.18 & Radix et Rhizoma Rhei \\
\hline Rheidin $\mathrm{A}^{*}$ & $\mathrm{C}_{30} \mathrm{H}_{22} \mathrm{O}_{8}$ & 510.49 & 1.24 & 0.58 & Radix et Rhizoma Rhei \\
\hline 3,4,5-Trihydroxybenzoic acid* & $\mathrm{C}_{7} \mathrm{H}_{6} \mathrm{O}_{5}$ & 170.12 & 31.69 & 0.04 & Radix et Rhizoma Rhei \\
\hline Mandenol & $\mathrm{C}_{20} \mathrm{H}_{36} \mathrm{O}_{2}$ & 308.50 & 42.00 & 0.19 & Pericarpium Trichosanthis \\
\hline Diosmetin & $\mathrm{C}_{16} \mathrm{H}_{12} \mathrm{O}_{6}$ & 300.26 & 31.14 & 0.27 & Pericarpium Trichosanthis \\
\hline Hydroxygenkwanin & $\mathrm{C}_{16} \mathrm{H}_{12} \mathrm{O}_{6}$ & 300.26 & 36.47 & 0.27 & Pericarpium Trichosanthis \\
\hline Schottenol & $\mathrm{C}_{29} \mathrm{H}_{50} \mathrm{O}$ & 414.70 & 37.42 & 0.75 & Pericarpium Trichosanthis \\
\hline $10 \alpha$-Cucurbita-5,24-diene-3 $\beta$-ol & $\mathrm{C}_{30} \mathrm{H}_{50} \mathrm{O}$ & 426.70 & 44.02 & 0.74 & Pericarpium Trichosanthis \\
\hline 7-Oxo-dihydrokaro-unidiol & $\mathrm{C}_{30} \mathrm{H}_{48} \mathrm{O}_{3}$ & 456.70 & 36.85 & 0.75 & Pericarpium Trichosanthis \\
\hline Linolenic acid ethyl ester & $\mathrm{C}_{20} \mathrm{H}_{34} \mathrm{O}_{2}$ & 306.50 & 46.10 & 0.20 & Pericarpium Trichosanthis \\
\hline Vitamin-e & $\mathrm{C}_{29} \mathrm{H}_{50} \mathrm{O}_{2}$ & 430.70 & 32.29 & 0.70 & Pericarpium Trichosanthis \\
\hline 4-Hydroxy-2-methoxybenzoic acid* & $\mathrm{C}_{8} \mathrm{H}_{8} \mathrm{O}_{3}$ & 152.15 & 50.76 & 0.04 & Pericarpium Trichosanthis \\
\hline Adenosine* & $\mathrm{C}_{10} \mathrm{H}_{13} \mathrm{~N}_{5} \mathrm{O}_{4}$ & 267.24 & 19.85 & 0.16 & Pericarpium Trichosanthis \\
\hline Luteolin-7-O-glucoside* & $\mathrm{C}_{21} \mathrm{H}_{20} \mathrm{O}_{11}$ & 448.40 & 7.29 & 0.78 & Pericarpium Trichosanthis \\
\hline Palmitoleic acid* & $\mathrm{C}_{16} \mathrm{H}_{30} \mathrm{O}_{2}$ & 254.41 & 35.78 & 0.10 & Pericarpium Trichosanthis \\
\hline Glycerol monopalmitate* & $\mathrm{C}_{19} \mathrm{H}_{38} \mathrm{O}_{4}$ & 330.50 & 20.43 & 0.43 & Pericarpium Trichosanthis \\
\hline Oxofangchirine* & $\mathrm{C}_{37} \mathrm{H}_{34} \mathrm{~N}_{2} \mathrm{O}_{7}$ & 618.70 & 20.61 & 0.33 & Pericarpium Trichosanthis \\
\hline Linoleyl acetate & $\mathrm{C}_{20} \mathrm{H}_{36} \mathrm{O}_{2}$ & 308.50 & 42.10 & 0.20 & Pericarpium Trichosanthis \\
\hline Estrone & $\mathrm{C}_{18} \mathrm{H}_{22} \mathrm{O}_{2}$ & 270.40 & 53.56 & 0.32 & Semen Armeniacae Amarum \\
\hline Diisooctyl succinate & $\mathrm{C}_{20} \mathrm{H}_{38} \mathrm{O}_{4}$ & 342.50 & 31.62 & 0.23 & Semen Armeniacae Amarum \\
\hline 11,14-Eicosadienoic acid & $\mathrm{C}_{20} \mathrm{H}_{36} \mathrm{O}_{2}$ & 308.50 & 39.99 & 0.20 & Semen Armeniacae Amarum \\
\hline Squalene & $\mathrm{C}_{30} \mathrm{H}_{50}$ & 410.70 & 33.55 & 0.42 & Semen Armeniacae Amarum \\
\hline Sitosterol & $\mathrm{C}_{29} \mathrm{H}_{50} \mathrm{O}$ & 414.70 & 36.91 & 0.75 & Semen Armeniacae Amarum \\
\hline Gondoic acid & $\mathrm{C}_{20} \mathrm{H}_{38} \mathrm{O}_{2}$ & 310.50 & 30.70 & 0.20 & Semen Armeniacae Amarum \\
\hline Cholesterol & $\mathrm{C}_{27} \mathrm{H}_{46} \mathrm{O}$ & 386.70 & 5.69 & 0.67 & Semen Armeniacae Amarum \\
\hline Mairin & $\mathrm{C}_{30} \mathrm{H}_{48} \mathrm{O}_{3}$ & 456.70 & 55.38 & 0.78 & Semen Armeniacae Amarum \\
\hline
\end{tabular}


Table 1 (continued)

\begin{tabular}{|c|c|c|c|c|c|}
\hline Molecular Name & Molecular Formula & Molecular Weight & $\mathrm{OB}(\%)$ & DL & Chinese medicine \\
\hline Catechin & $\mathrm{C}_{15} \mathrm{H}_{14} \mathrm{O}_{6}$ & 290.27 & 54.83 & 0.24 & Semen Armeniacae Amarum \\
\hline Glycyrol & $\mathrm{C}_{21} \mathrm{H}_{18} \mathrm{O}_{6}$ & 366.40 & 90.78 & 0.67 & Semen Armeniacae Amarum \\
\hline Licochalcone B & $\mathrm{C}_{16} \mathrm{H}_{14} \mathrm{O}_{5}$ & 286.28 & 76.76 & 0.19 & Semen Armeniacae Amarum \\
\hline Liquiritin & $\mathrm{C}_{21} \mathrm{H}_{22} \mathrm{O}_{9}$ & 418.40 & 65.69 & 0.74 & Semen Armeniacae Amarum \\
\hline Glabridin & $\mathrm{C}_{20} \mathrm{H}_{20} \mathrm{O}_{4}$ & 324.40 & 53.25 & 0.47 & Semen Armeniacae Amarum \\
\hline Machiline & $\mathrm{C}_{17} \mathrm{H}_{19} \mathrm{NO}_{3}$ & 285.34 & 79.64 & 0.24 & Semen Armeniacae Amarum \\
\hline Stepholidine & $\mathrm{C}_{19} \mathrm{H}_{21} \mathrm{NO}_{4}$ & 327.40 & 33.11 & 0.54 & Semen Armeniacae Amarum \\
\hline Amygdalin* & $\mathrm{C}_{20} \mathrm{H}_{27} \mathrm{NO}_{11}$ & 457.40 & 4.42 & 0.61 & Semen Armeniacae Amarum \\
\hline Spinasterol & $\mathrm{C}_{29} \mathrm{H}_{48} \mathrm{O}$ & 412.70 & 42.98 & 0.76 & $\begin{array}{l}\text { Pericarpium Trichosanthis } \\
\text { Semen Armeniacae Amarum }\end{array}$ \\
\hline Stigmasterol & $\mathrm{C}_{29} \mathrm{H}_{48} \mathrm{O}$ & 412.70 & 43.83 & 0.76 & $\begin{array}{l}\text { Pericarpium Trichosanthis } \\
\text { Semen Armeniacae Amarum }\end{array}$ \\
\hline Calcium sulfate dihydrate & $\mathrm{CaSO}_{4} \cdot 2 \mathrm{H}_{2} \mathrm{O}$ & 172.17 & - & - & Gypsum Fibrosum \\
\hline
\end{tabular}

*The compound from literature mining

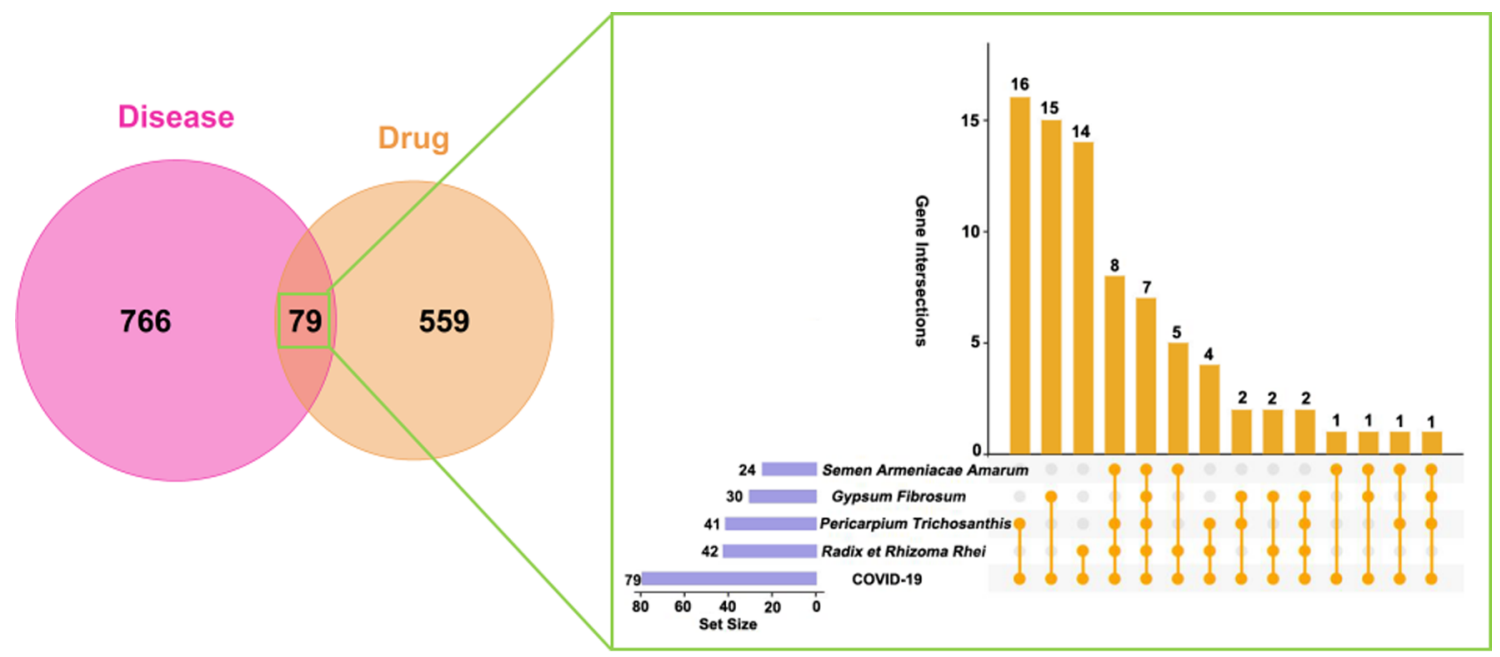

$\mathbf{a}$

Fig. 3 Intersection analysis between COVID-19 disease targets and compounds targets of XBCQD; a Venn diagram for intersection analysis of compound targets and disease targets; $\mathbf{b}$ Upset plot of targets among 4 Chinese medicines of XBCQD and COVID-19. The hori-

To find the functional connections of the clusters in the PPI network, the MCODE module of Cytoscape 3.7.2 software was employed to analyze the 67 crucial targets of the PPI network. 4 submodules were identified, covering 26 out of the 67 targets (Fig. 4b). Module 1 (score: 5.273) was composed of 12 nodes and 29 edges, and the seed gene was PRKCB. Module 2 (score: 4.000) was composed of 8 nodes and 14 edges, and the seed gene was PIK3CA. Module 3 (score: 3.000 ) consisted of 3 nodes and 3 edges, and the seed gene was VCP. Module 4 (score: 3.000 ) consisted of 3 nodes and 3 edges, and the seed gene was TLR7. Then GO enrichment analysis was performed to investigate the b

zontal bar graph at the bottom left shows the total number of targets for each part. Circles and vertical lines on the x-axis show the correlation between the parts being compared. The vertical bar graph at the top shows the number of targets in the comparisons

biological process of 4 submodules (Fig. 4c). The results showed that these 4 modules had anti-inflammatory, antiviral, anti-tumor, and immune regulation effects.

\section{Construction of the Chinese medicines-effective compounds-hub targets (C-E-H) network}

In this section, we used Cytoscape 3.7.2 software to construct a C-E-H network to obtain the effective compounds, and employed the NetworkAnalyzer plugin to analyze its topology properties. In Fig. 5, there were 87 nodes, including 4 Chinese medicines, 49 effective compounds and 34 
Table 279 putative targets of XBCQD for the treatment of COVID-19

\begin{tabular}{llllllll}
\hline No. & Gene & No. & Gene & No. & Gene & No. & Gene \\
\hline 1 & ACE2 & 21 & LGALS3 & 41 & CCL5 & 61 & GAPDH \\
2 & DPP4 & 22 & F3 & 42 & MAPK3 & 62 & PRKCE \\
3 & CTSL & 23 & ABO & 43 & PARP1 & 63 & F10 \\
4 & IL6 & 24 & JAK1 & 44 & BAX & 64 & PRKCA \\
5 & CTSB & 25 & SREBF2 & 45 & BCL2 & 65 & APOBEC3G \\
6 & COMT & 26 & TNF & 46 & PIK3CA & 66 & GSK3A \\
7 & PRKACA & 27 & TGFB1 & 47 & CASP8 & 67 & EIF4E \\
8 & DNMT1 & 28 & NFkB1 & 48 & CDK2 & 68 & PTGS1 \\
9 & PLAT & 29 & JAK2 & 49 & PTGS2 & 69 & TTR \\
10 & POR & 30 & F2 & 50 & NOS2 & 70 & IGKC \\
11 & CSNK2B & 31 & CASP3 & 51 & PIK3CB & 71 & LCK \\
12 & IMPDH2 & 32 & MBL2 & 52 & MCL1 & 72 & LCN2 \\
13 & POLA1 & 33 & IL1A & 53 & CREB1 & 73 & BCL2A1 \\
14 & SIGMAR1 & 34 & IL2 & 54 & SIRT1 & 74 & CALM1 \\
15 & SIRT5 & 35 & TLR7 & 55 & RELA & 75 & NAE1 \\
16 & PRKAR2B & 36 & ADA & 56 & HSP90B1 & 76 & PRSS2 \\
17 & AR & 37 & CSF2 & 57 & PIK3CG & 77 & PRKCB \\
18 & IL1B & 38 & ANPEP & 58 & ACTB & 78 & ENPP1 \\
19 & NOS3 & 39 & PPARG & 59 & GSK3B & 79 & HPGDS \\
20 & CCL2 & 40 & TP53 & 60 & VCP & & \\
\hline
\end{tabular}

hub targets. The size of the nodes were proportional to the degree values. The larger the node, the higher the degree value. The complex network showed that one compound in XBCQD could interact with multiple targets, and one target could be regulated by multiple components. For example, epicatechin in Radix et Rhizoma Rhei targeted on 10 hub targets, including PTGS2, TNF, IL6, IL1A, PPKACA, CASP3, JAK1, CCL2, CREB1 and IL2. Emodin could also regulate 9 hub targets, such as PTGS2, TNF, TP53, PPKACA, PPARG, IL1B, CSF2, PPKCE and CASP3. There also have other compounds and related targets, which reflect the positive characteristics of multi-component and multi-target intervention of XBCQD in the treatment of COVID-19. Since calcium sulfate dihydrate $\left(\mathrm{CaSO}_{4} \cdot 2 \mathrm{H}_{2} \mathrm{O}\right)$ is an inorganic salt, we focused on other effective compounds for analysis. Finally, the C-E-H network revealed that 18 effective compounds had higher degree values than the median degree value of 3 , indicating that these core compounds had a significant therapeutic effect on COVID-19, such as epicatechin, emodin, aloe-emodin, adenosine, glabridin, licochalcone B, glycyrol, eupatin, diosmetin, luteolin-7-O-glucoside, (-)-catechin, hydroxygenkwanin, stigmasterol, spinasterol, estrone, gondoic acid, 11,14-eicosadienoic acid and betasitosterol [53, 54].

\section{GO function and KEGG pathway enrichment analysis}

In order to more intuitively explain the therapeutic mechanism of XBCQD in the treatment of COVID-19, we used
R 3.6.3 software to perform GO annotation analysis and KEGG pathway analysis on 34 hub targets. After filtering with $p$ value $\leq 0.05$, a total of $1639 \mathrm{GO}$ terms were included, of which 1538 for biological processes (BP), 83 for molecular functions (MF), and 18 for cellular components (CC). The enrichment results were saved and sorted according to the $p$ value of each entry into the selected top biological processes and pathways. As shown in Fig. 6a, the enrichment results of $\mathrm{CC}$ and MF revealed that the target genes were mainly expressed in the membrane rafts, membrane microdomains and other cell compartments, and were related to cytokine receptor binding, ubiquitin protein ligase binding and growth factor receptor binding. In terms of BP, apoptosis obviously played a confrontational role in the treatment of COVID-19, in which extrinsic apoptotic, regulation of apoptotic and negative regulation of apoptotic process were significantly enriched. In addition, signal transduction and cellular response were also associated with COVID-19.

KEGG pathway enrichment analysis provided further information about gene functions and their interactions. The results revealed that 180 KEGG pathway terms were obtained through the enrichment of 34 hub targets, with $p$ value $\leq 0.05$. As shown in Fig. $6 \mathrm{~b}$, the hub targets mainly participated in pathways associated with viral infection and inflammatory injury, including AGE-RAGE signaling pathway, Hepatitis B, TNF signaling pathway, IL-17 signaling pathway and so on. Among these signaling pathways, there were 14 targets (RELA, MAPK3, NFkB1, PIK3CA, IL6, TNF, IL1B, CASP8, CSF2, CREB1, PIK3CB, CASP3, 


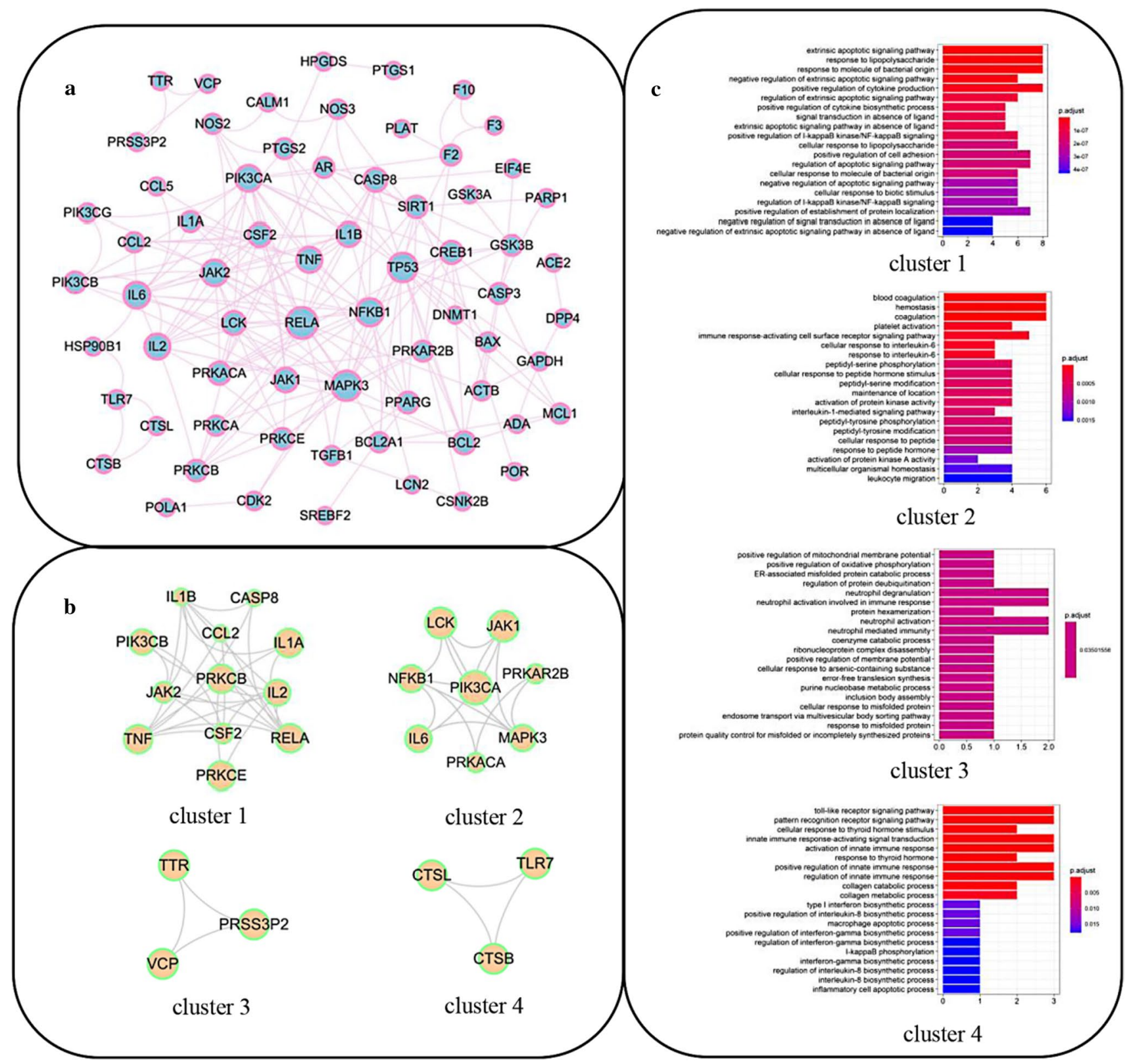

Fig. 4 Correlation analysis of crucial targets of intersection targets; a PPI network of crucial targets for XBCQD against COVID-19; $\mathbf{b}$ MCODE analysis of PPI network; c Biological process analysis of 4 submodules

PTGS2 and CCL2) participating in the TNF signaling pathway, 12 targets (RELA, MAPK3, NFkB1, IL6, TNF, IL1B, CASP8, CSF2, CASP3, GSK3B, PTGS2 and CCL2) participating in IL-17 signaling pathway, 16 targets (RELA, MAPK3, NFкB1, PIK3CA, IL6, JAK2, TNF, IL1B, BCL2, PIK3CB, PRKCE, PRKCB, CASP3, CCL2, IL1A and PRKCA) participating in the AGE-RAGE signaling pathway, 16 targets (RELA, MAPK3, TP53, NFкB1, PIK3CA, IL6, JAK2, TNF, JAK1, CASP8, BCL2, CREB1, PIK3CB, PRKCB, CASP3 and PRKCA) participating in the Hepatitis B, respectively. IL-17 signaling pathway is an important inflammatory pathway. IL-17 ligand binds and activates the corresponding receptor, and then the complex recruits ACT1 from the cytoplasm through the SEFIR domain. ACT1 initiates TNF receptor-associated factor 6 (TRAF6) to activate $\mathrm{NF}-\kappa \mathrm{B}$ signaling pathway, thereby improving the expression levels of pro-inflammatory factors [55]. According to current research, COVID-19 patients usually have an elevated rise in the cytokine levels, which were associated with significantly worse health conditions in those infected [56]. Therefore, we speculated that the activation of IL-17 signaling pathway was closely related to the development of COVID-19. 


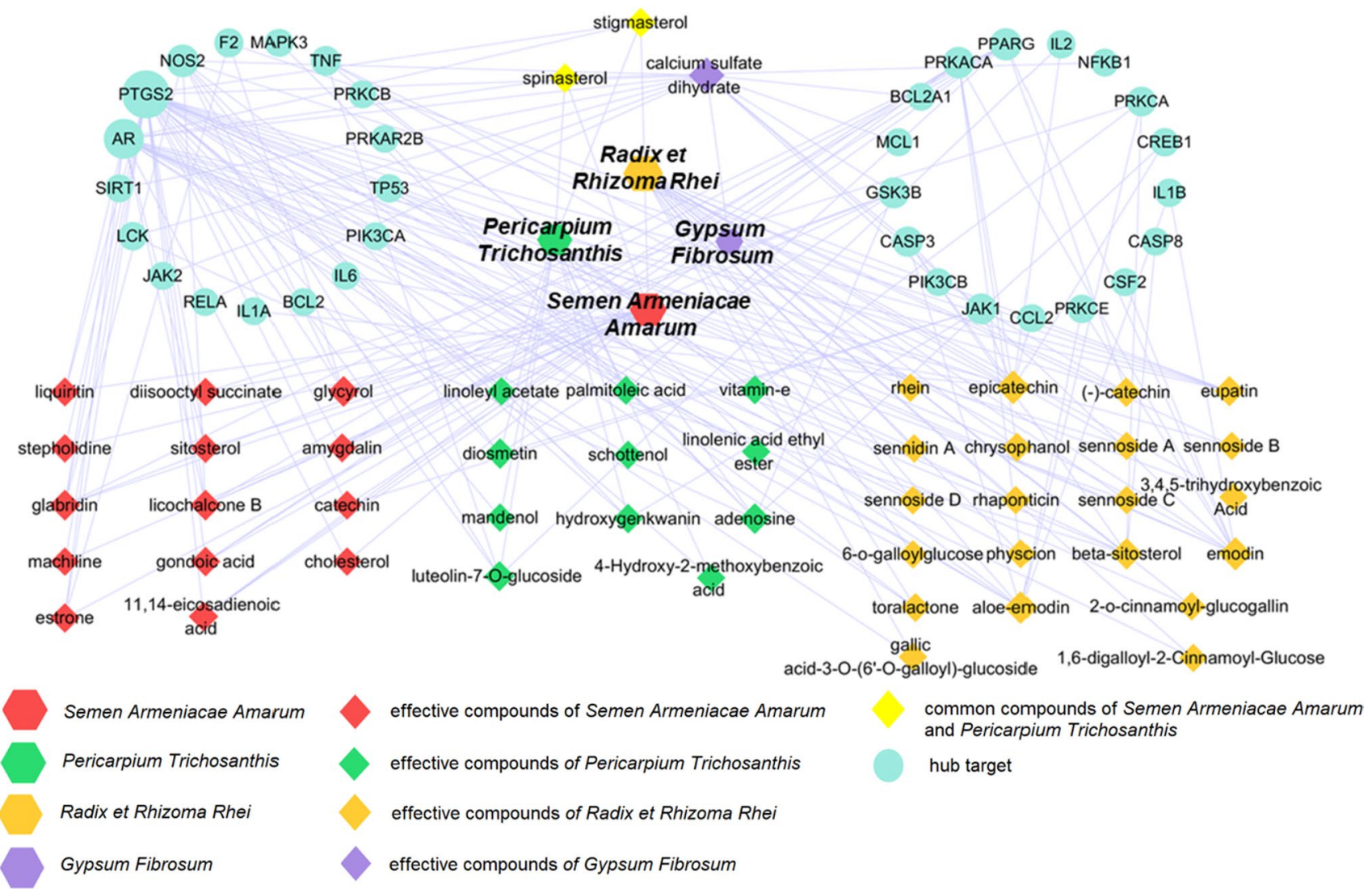

Fig. 5 The C-E-H network of XBCQD against COVID-19. The red, green, orange, and purple hexagons represented Chinese medicines as Semen Armeniacae Amarum, Pericarpium Trichosanthis, Radix et Rhizoma Rhei and Gypsum Fibrosum. The red, green, orange, and purple rhombuses represented effective compounds of the above

Moreover, we found that several hub targets could simultaneously act on multiple pathways, which indicated the importance of specific targets in the whole disease bioinformatics network, and changed in these targets may affect the entire network to produce a chain reaction. For example, RELA, NFkB1 and IL6 were significantly enriched in all 9 pathways, as well as PIK3CA appeared in 8 pathways.

\section{Drug-likeness analysis and ADMET evaluation of the selected compounds}

Drug-likeness assessment is a key factor in screening compounds with drug-like properties. The molecular properties include molecular weight, Van der Waals volume, number of hydrogen bond acceptors (nHA), number of hydrogen bond donors (nHD), topological polar surface area (TPSA), number of rotatable bonds (nRot), number of rings (nRing), Log of the aqueous solubility $(\log S), \log$ of the octanol/water partition coefficient $(\log \mathrm{P})$, and $\log \mathrm{P}$ at physiological $\mathrm{pH}$ $7.4(\log D)$. According to the Lipinski's rule of five, Pfizer rule and Golden triangle rule, 10 of the 18 core compounds
Chinese medicines. Yellow rhombuses represented the common compounds of Semen Armeniacae Amarum and Pericarpium Trichosanthis. Cyan circles represented hub targets. The size of the node represented degree value. The line between two nodes represented the interaction

satisfied all the filtration criteria, such as epicatechin, emodin, aloe-emodin, adenosine, licochalcone B, glycyrol, eupatin, diosmetin, (-)-catechin, and hydroxygenkwaninas shown in Table $\mathrm{S} 2$.

The ADMET evaluation of the pharmacokinetic properties of these selected 10 core compounds were described in Table S3. Licochalcone B and eupatin have high Caco-2 permeability and high oral bioavailability indicated by F30\% than the others. Intestinal absorption analysis (HIA) revealed good absorption of all compounds except aloe-emodin and adenosine. Epicatechin, emodin, aloe-emodin, licochalcone $\mathrm{B}$ and (-)-catechin were non-substrates or non-inhibitors of P-glycoprotein, other compounds were substrates or inhibitors of P-glycoprotein. With the exception of adenosine, almost all selected compounds could penetrate the blood brain barrier (BBB). Epicatechin, adenosine and (-)-catechin were all non-inhibitors of cytochrome P450s (CYP). Emodin, aloe-emodin, licochalcone B, glycyrol, eupatin, diosmetin, and hydroxygenkwanin showed inhibitory activities on CYP1A2 and CYP2C9 enzymes. Besides, aloe-emodin, adenosine and eupatin showed lower clearance (CL) and 


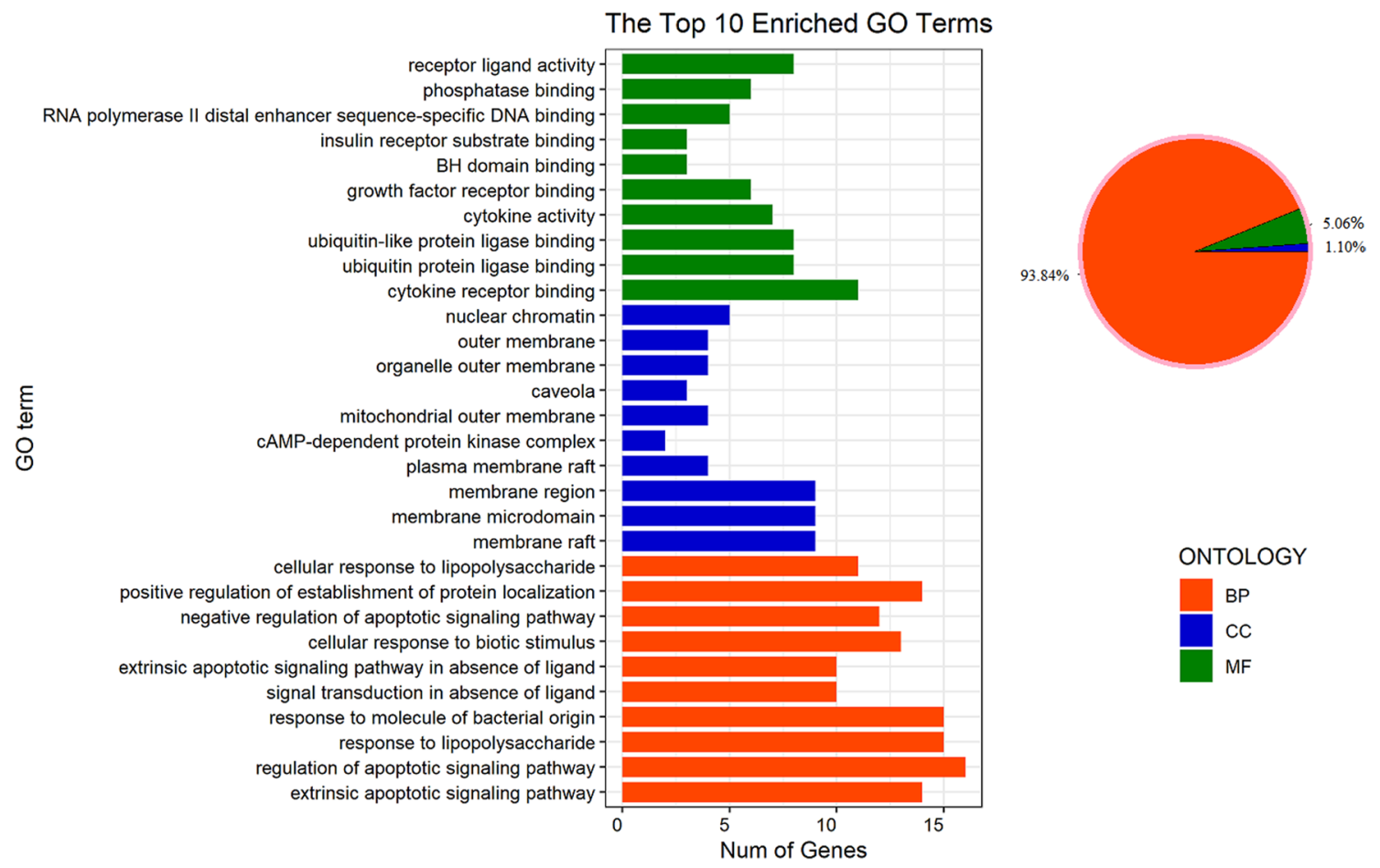

a

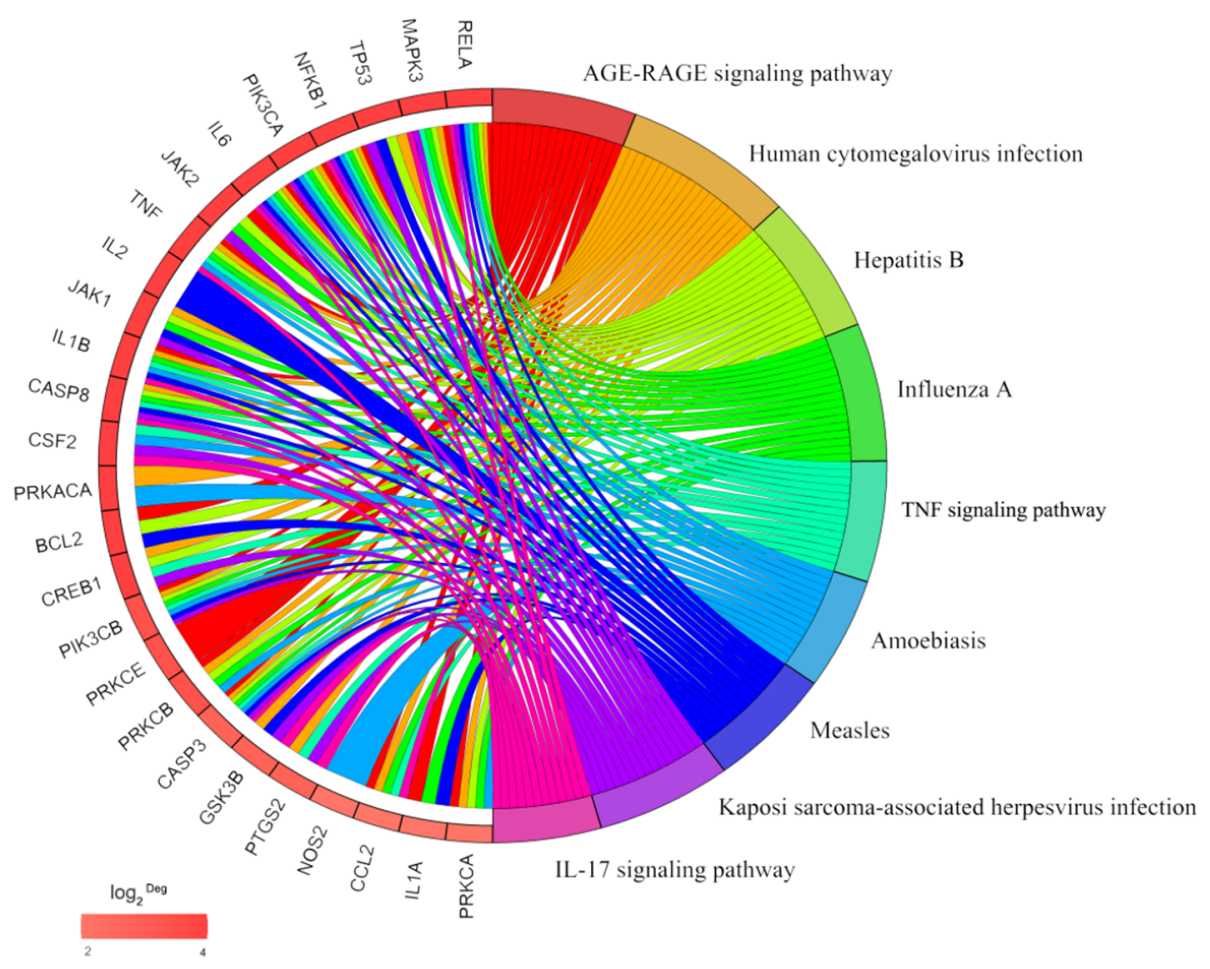

b

Fig. $6 \mathrm{GO}$ and KEGG enrichment analysis. a Bar plot showed the top $10 \mathrm{GO}$ enrichment terms of BP (orange), CC (blue) and MF (green), respectively. The pie plot showed the proportion of each part of BP, $\mathrm{CC}$ and MF to the whole; $\mathbf{b}$ The chord plot showed the top 9 KEGG pathway terms and corresponding targets. The right half of the chord plot referred to the enrichment pathway, and the left half referred to the genes targeted by the pathway. $\log _{2}$ Deg was equal to $\log _{2}$ Degree, which reflected the size of the degree value 
longer half-life $\left(\mathrm{T}_{1 / 2}\right)$ than other compounds. All $10 \mathrm{com}-$ pounds were not hERG blockers. Adenosine and glycyrol showed some human hepatotoxicity (H-HT). Aloe-emodin, adenosine, and licochalcone $\mathrm{B}$ were predicted to have a little respiratory toxicity. Only licochalcone B showed possible carcinogenicity toxicity. However, almost all the selected core compounds showed lower toxicity than the reference drugs remdesivir and telaprevir.

\section{Molecular docking analysis}

Molecular docking was performed by Sybyl-X 2.1.1 software to evaluate the binding affinity between ligand and receptor. We selected the targets with degree greater than 9.5 (average degree) from 34 hub targets as protein receptors. Due to lack of applicable ligands of a few targets in the PDB, the target of MAPK3 (PDB ID: 2ZOQ), TP53 (PDB ID: 5AOK), PIK3CA (PDB ID: 6GVF), JAK2 (PDB ID: 3FUP), TNF (PDB ID: 6X81), IL2 (PDB ID: 1NBP), JAK1 (PDB ID: 4K6Z), IL1B (PDB ID: 5R87), CASP8 (PDB ID:3KJQ) and LCK (PDB ID: 2ZM1) were selected to construct proteins receptor. The co-crystallized ligands or inhibitors used as the reference ligands were first redocked to the protein's binding site, respectively, to set a threshold binding affinity value for comparing the results with other compounds. Then, we selected 10 core compounds that passed all the screening criteria for drug-likeness assessment, namely epicatechin, emodin, aloe-emodin, adenosine, licochalcone B, glycyrol, eupatin, diosmetin, (-)-catechin, and hydroxygenkwaninas for molecular docking validation. Moreover, we also selected these core compounds to dock with SARS-CoV-2 3CL protease (PDB ID: 6WTT) and ACE2(PDB ID: 1R4L), which are effective targets for the treatment of COVID-19 infection [57-60].

As shown in Fig. 7, almost all the core compounds exhibited effective affinity for their hub targets contrasted to the reference ligands. Figure 8 shows the 3D diagrams of binding mode between representative core compounds and related hub targets. Compared with the reference ligands, the core compounds made the expected interaction with residues of acitive binding sites and exhibited high degree of geometric complementarity. For the binding mode between hydroxygenkwanin and $3 \mathrm{CL}$ protease (Fig. 8a), the residues of Met49, Tyr54, Gly143, Glu166, Asp187 and Gln189 formed hydrogen bond interactions with hydroxygenkwanin, indicating that hydroxygenkwanin bound to the active site of

\section{Total score}

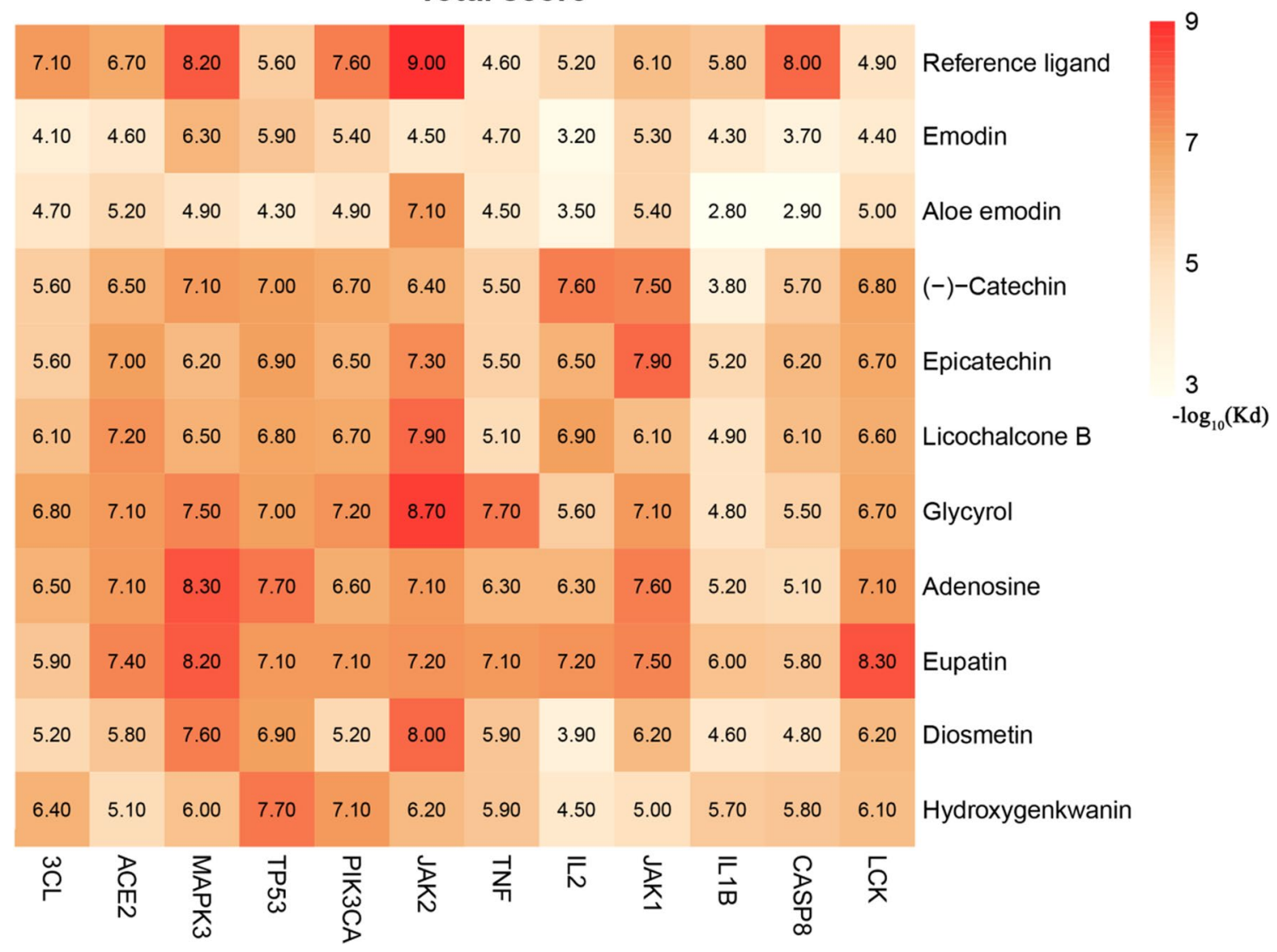

Fig. 7 The docking scores between core compounds and hub targets. Total scores are expressed in $-\log _{10}(\mathrm{Kd})$ units 


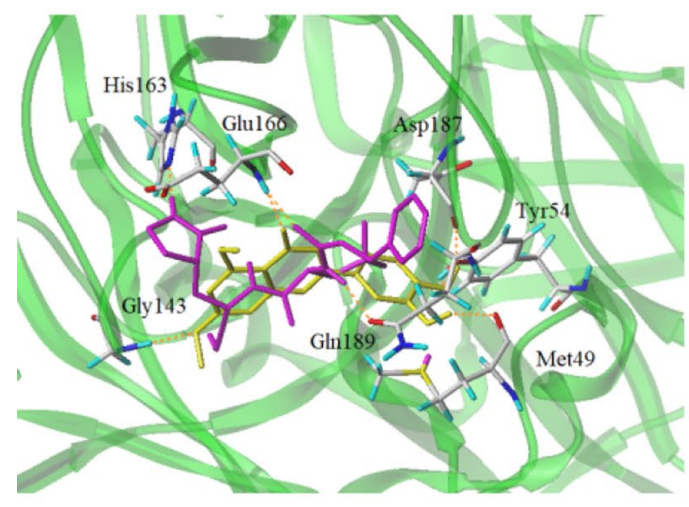

a

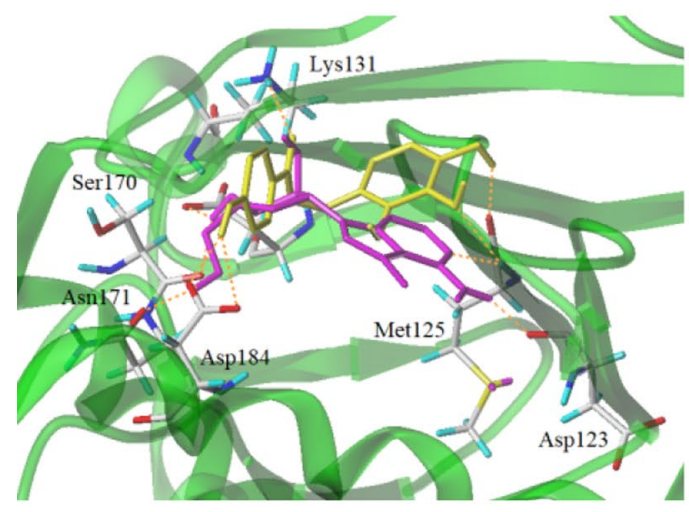

c

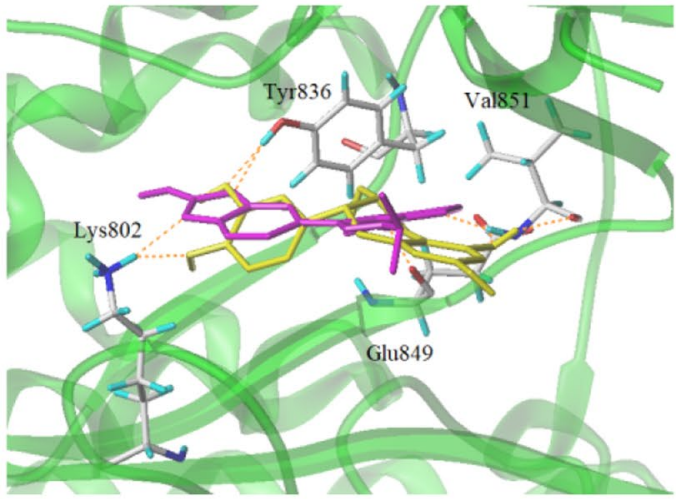

e

Fig. 8 The binding mode and conformation plots between representative core compounds and amino acid residues of hub targets. Magenta represents co-crystallized ligand or inhibitor as reference ligand. Yellow and green represent core compounds of XBCQD. Orange dashed

the 3CL successfully. Eupatin formed seven hydrogen bonds with Ala348, Thr371, His374, His378, Glu402, Tyr515 and Arg518 to enhance its stability with ACE2 (Fig. 8b). The residues of Met125, Lys131 and Asp184 in MAPK3 formed hydrogen bonds with licochalcone B (Fig. 8c). Figure 8d shows the docking diagram of glycyrol and TP53. Two hydrogen bonds were formed between glycyrol and Val147

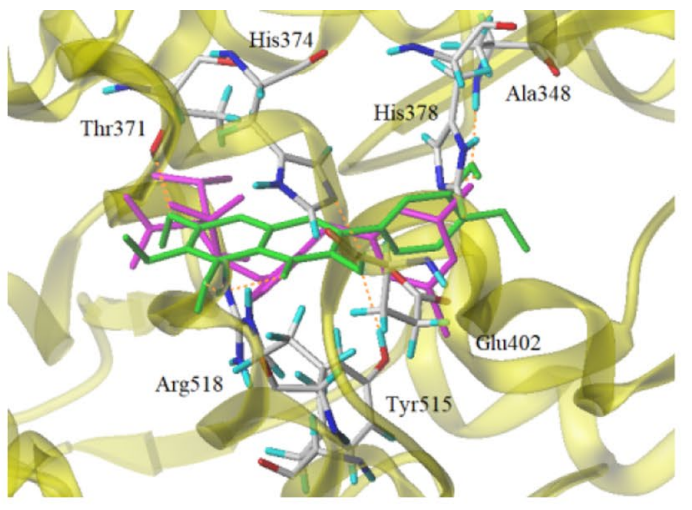

b

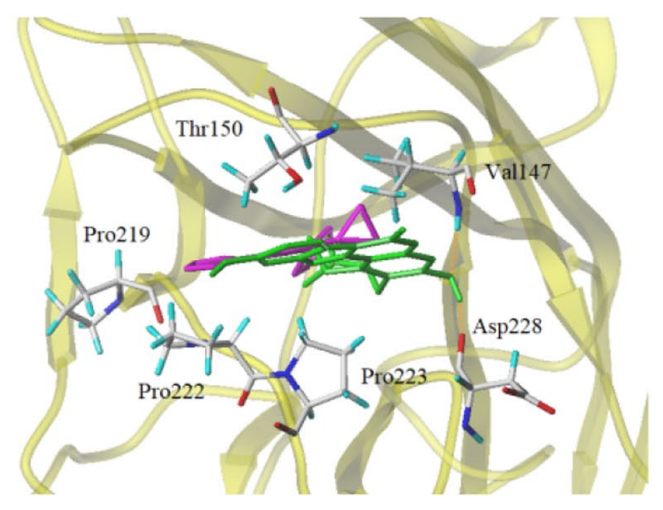

d

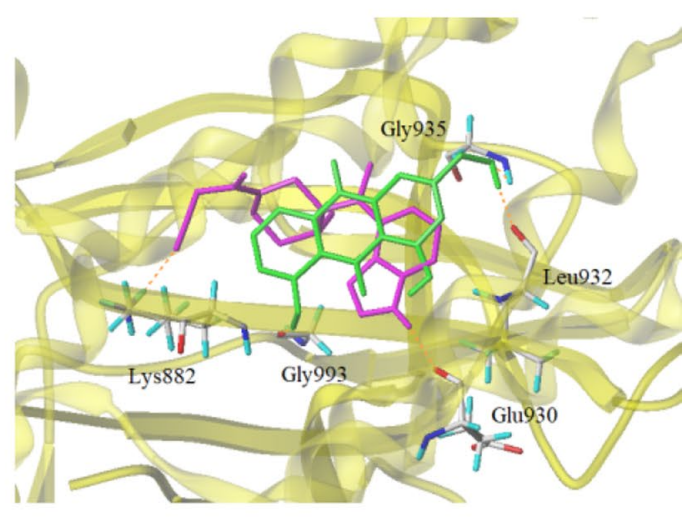

f

line represents hydrogen bond. a 3CL and hydroxygenkwanin; b ACE2 and eupatin; c MAPK3 and licochalcone B; d TP53 and glycyrol; e PIK3CA and epicatechin; f JAK2 and aloe-emodin

and Asp228, which enhances the stability of the conformation of glycyrol and TP53. As shown in Fig. 8e, epicatechin displayed a good binding ability with PIK3CA. Lys802, Tyr836 and Val851 formed hydrogen bond interactions with epicatechin to maintain conformational stability. The results shown in Fig. $8 \mathrm{f}$ indicated that aloe-emodin bound to JAK2 by generating three hydrogen bond with the surrounding 
residues, including Leu932, Gly935 and Gly993. According to our results, the compounds in XBCQD could act on the targets of COVID-19, which indicated that XBCQD has a certain positive effect in the treatment of COVID-19.

\section{Molecular dynamic (MD) simulation results}

To analyze the conformational dynamics of the above representative core compounds and targets, $100 \mathrm{~ns}$ MD simulations were performed for each complex and compared with the corresponding reference ligand. The RMSDs of the backbone atoms of all complex structures relative to the initial structures were compared to investigate their equilibrium and stability levels throughout the simulations, as shown in Fig. 9. The 3CL-Hydroxygenkwanin complex was stable at an average of about $0.27 \mathrm{~nm}$ till $90 \mathrm{~ns}$, then abruptly rose to about $0.4 \mathrm{~nm}$, and finally stabilized to $100 \mathrm{~ns}$. The ACE2Eupatin complex showed a minor upward trend from 0.4 to $0.6 \mathrm{~nm}$ at $15 \mathrm{~ns}$, followed by a stable equilibrium throughout the simulation. The MAPK3-Licochalcone B complex stabilized at about $0.5 \mathrm{~nm}$ during $100 \mathrm{~ns}$ of MD simulation. TP53-Glycyrol complex maintained an average RMSD of $0.2 \mathrm{~nm}$ untill $70 \mathrm{~ns}$, then showed a gradual rise to $0.4 \mathrm{~nm}$ at $80 \mathrm{~ns}$, and continued to the end. PIK3CA-Epicatechin complex rose from 0.5 to $0.7 \mathrm{~nm}$ around $20 \mathrm{~ns}$, then decreased rapidly and remained stable at $0.5 \mathrm{~nm}$ till $100 \mathrm{~ns}$, with a small drop to about $0.4 \mathrm{~nm}$ observed at $90 \mathrm{~ns}$. The JAK2Aloe emodin complex underwent fluctuations untill the end of the $100 \mathrm{~ns}$ period, with the mean RMSD remaining at $0.2 \mathrm{~nm}$.
The RMSF of each residue of the complex was calculated to evaluate the flexibility of residues that contributed to the structural fluctuations. The results showed that all representative compounds exhibited similar trends compared to the corresponding reference ligands, that was, they caused a certain degree of fluctuations in the same region of the proteins. The RMSF plots showed that the 3CL-Hydroxygenkwanin and MAPK3-Licochalcone B complexes had largest fluctuations at the protein terminal residues (Fig. 10a, c). Only a few residues of ACE2-Eupatin, TP53-Glycyrol, PIK3CA-Epicatechin and JAK2-Aloe emodin complexes showed higher fluctuations, indicating the atomic flexibility of loops (Fig. 10b, d-f). Overall, all complexes showed equilibrium fluctuations during the simulation.

To further understand the structural stability of the complexes, we determined the compactness of the protein structure by computing the Rg (Fig. 11). The Rg plots showed that the structural dynamics of 3CL-Hydroxygenkwanin, MAPK3-Licochalcone B, TP53-Glycyrol and JAK2-Aloe emodin complexes remained fairly stable throughout the simulation time, with the average $\mathrm{Rg}$ values of $1.57,1.76$, 1.67 and $1.58 \mathrm{~nm}$, respectively. A slight deviation in the Rg plot of ACE2-Eupatin and PIK3CA-Epicatechin can be seen during the simulation, and the steady equilibrium were finally observed at $100 \mathrm{~ns}$, with mean $\mathrm{Rg}$ values of 1.95 and $1.49 \mathrm{~nm}$, respectively.

H-bonds contribute significantly to stabilizing protein-ligand complexes. To understand the binding affinity of ligands to proteins, H-bonds interaction analysis was performed on MD trajectories to calculate the total number of H-bonds formed between protein-ligand complexes

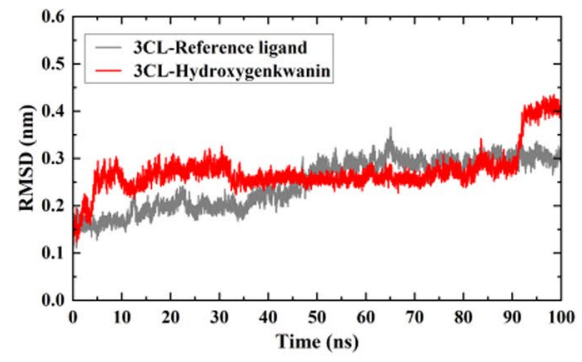

a

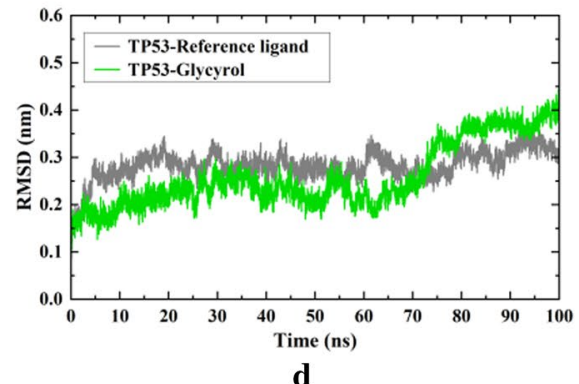

d

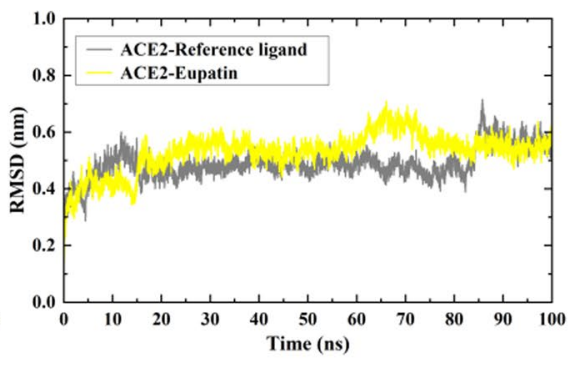

b

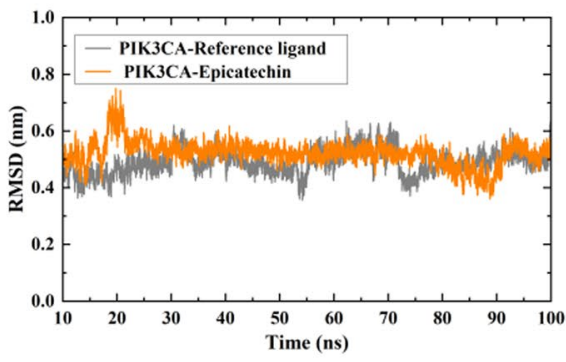

e

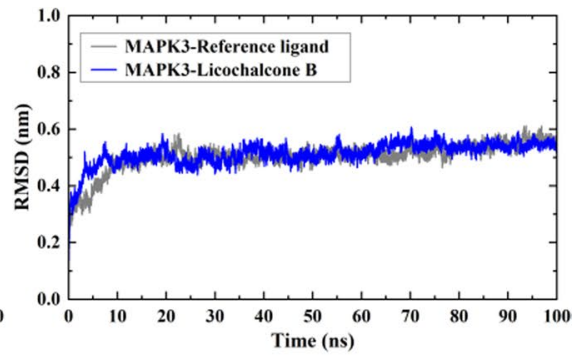

c

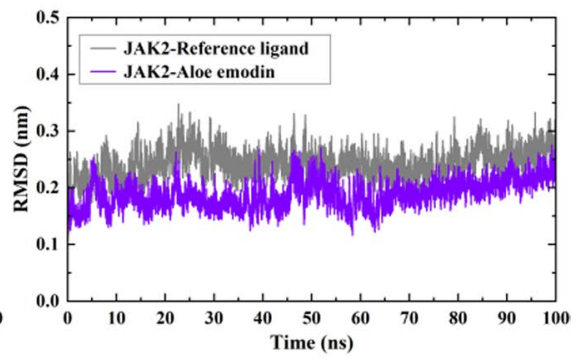

f

Fig. 9 RMSD trajectories values of complexes in the $100 \mathrm{~ns}$ period of MD simulations 


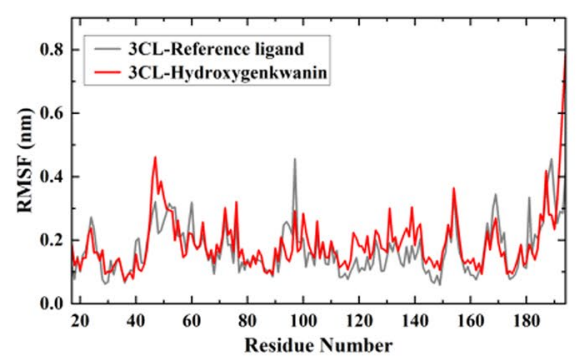

a

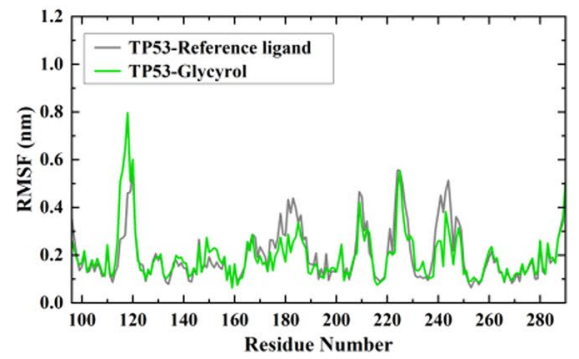

d

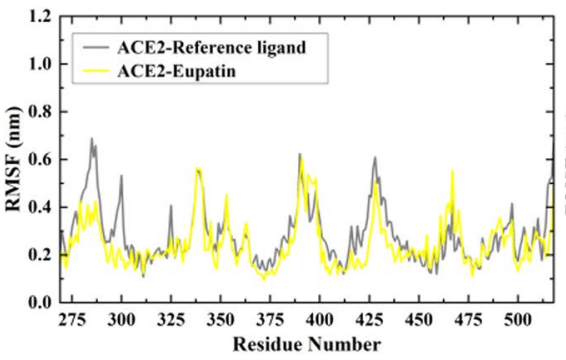

b

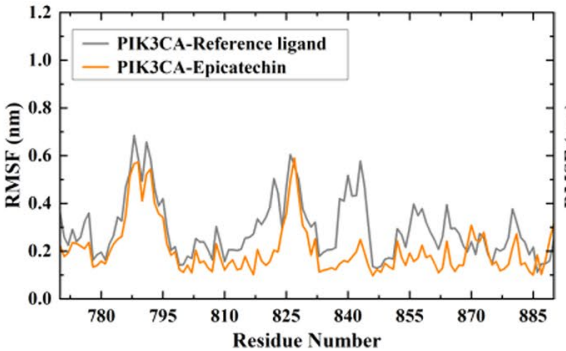

e

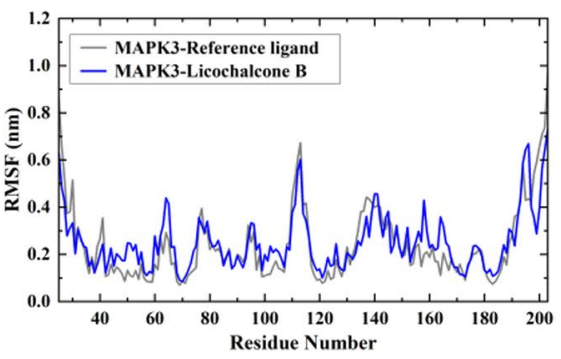

c

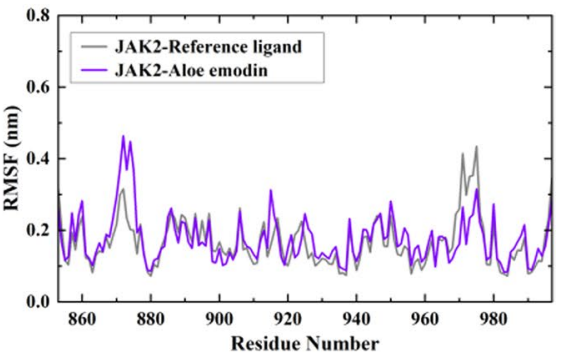

f

Fig. 10 RMSF trajectories values of complexes in the $100 \mathrm{~ns}$ period of MD simulations

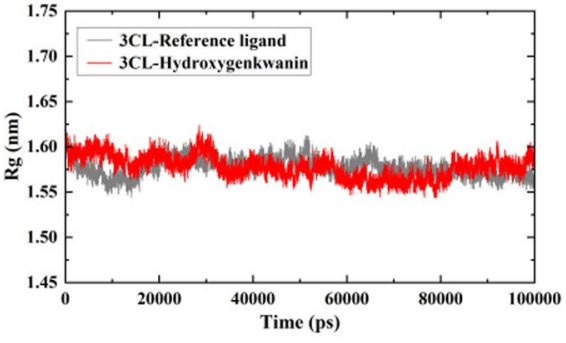

a

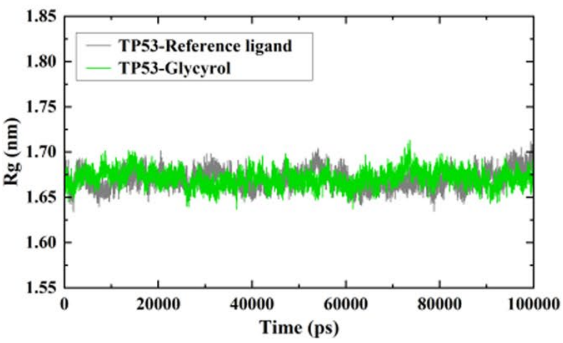

d

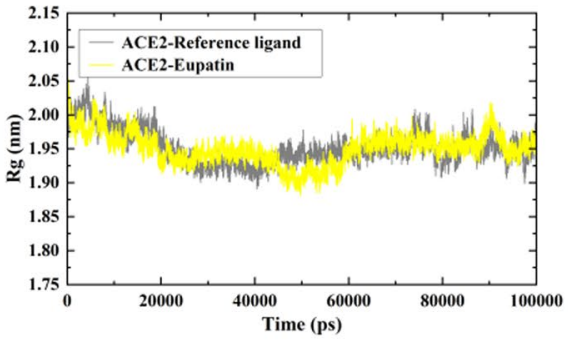

b

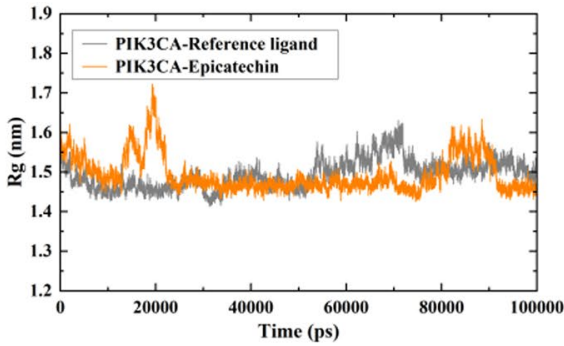

e

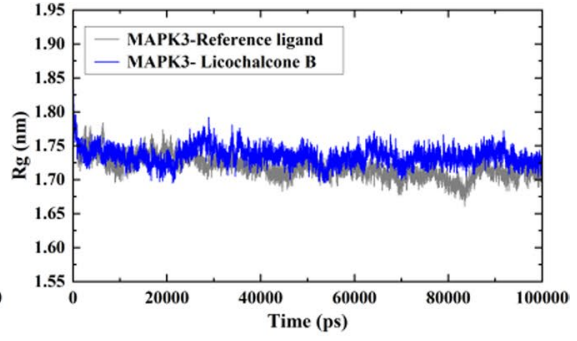

c

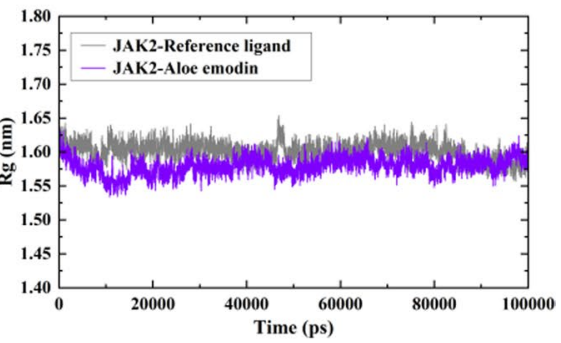

f

Fig. $11 \mathrm{Rg}$ trajectories values of complexes in the $100 \mathrm{~ns}$ period of MD simulations

(Fig. 12). The 3CL-Hydroxygenkwanin, ACE2-Eupatin, MAPK3-Licochalcone B, TP53-Glycyrol, PIK3CA-Epicatechin and JAK2-Aloe emodin complexes showed the number of $\mathrm{H}$-bonds to be between $0-5,2-10,0-9,0-5$, $0-8$ and $0-6$, respectively. The results suggested that the number of $\mathrm{H}$-bonds formed by all protein-ligand complexes was stable throughout the simulations. The amino acid residues in the active site were also persistent, helping to stabilize the entire complex structure.

The binding free energies of the complexes were calculated using the MM-PBSA method. As shown in Table 3, the free binding energy of TP53-glycyrrhizin complex was the largest at $-138.106 \mathrm{~kJ} / \mathrm{mol}$, followed by PIK3CAEpicatechin, 3CL-Hydroxygenkwanin, ACE2-Eupatin, 


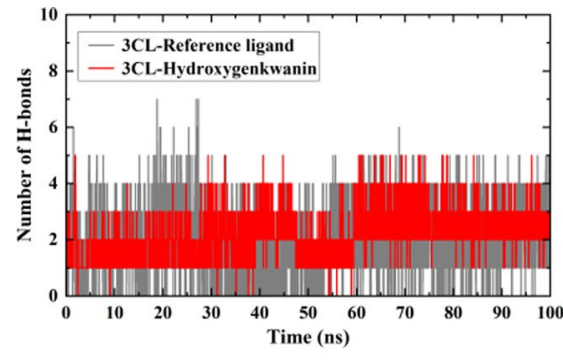

a

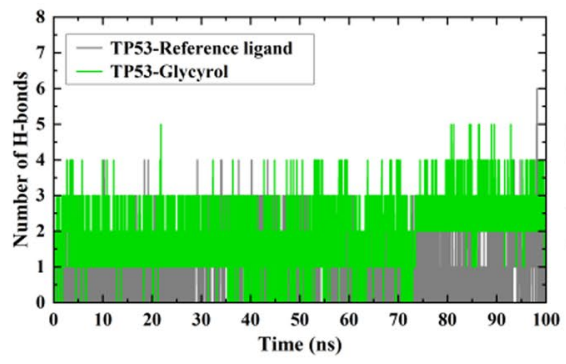

d

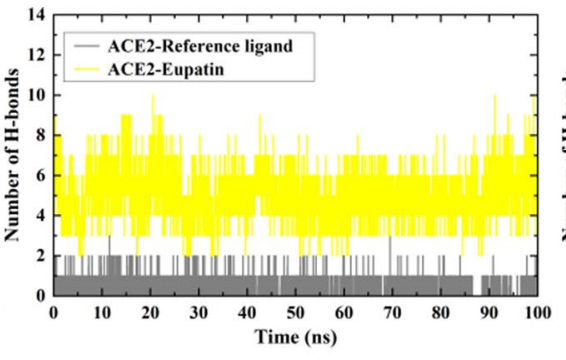

b

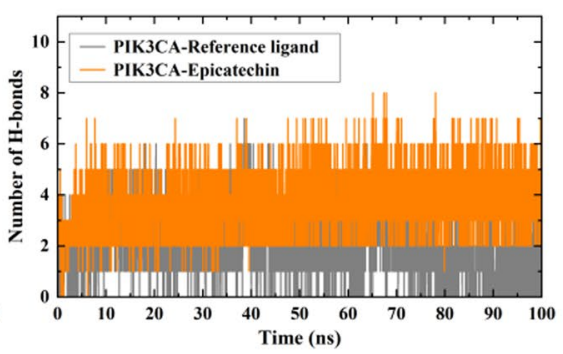

e

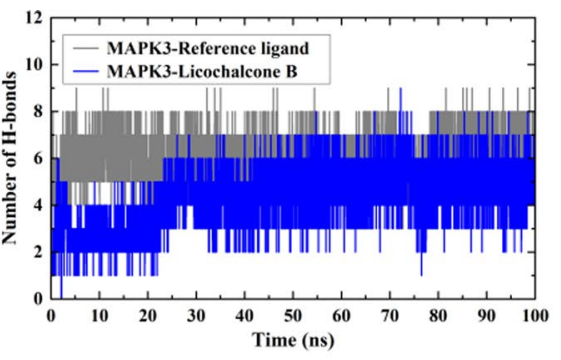

c

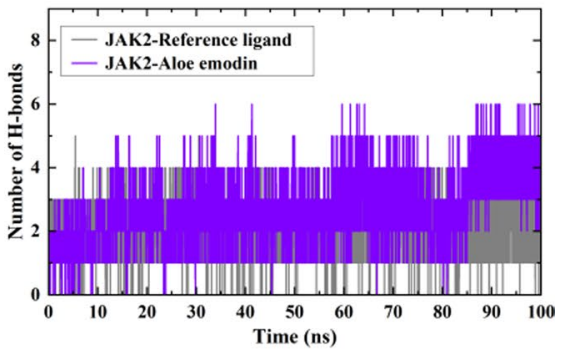

f

Fig. 12 The number of hydrogen bonds for complex systems in the $100 \mathrm{~ns}$ period of MD simulations

Table 3 Calculated binding free energy values with MM-PBSA method

\begin{tabular}{llllll}
\hline & $\begin{array}{l}\text { Van der Waal energy } \\
(\mathrm{kJ} / \mathrm{mol})\end{array}$ & $\begin{array}{l}\text { Electrostattic energy } \\
(\mathrm{kJ} / \mathrm{mol})\end{array}$ & $\begin{array}{l}\text { Polar solvation } \\
\text { energy }(\mathrm{kJ} / \mathrm{mol})\end{array}$ & SASA energy $(\mathrm{kJ} / \mathrm{mol})$ & Binding energy $(\mathrm{kJ} / \mathrm{mol})$ \\
\hline $\begin{array}{l}\text { 3CL-Hydroxygenk- } \\
\text { wanin }\end{array}$ & $-127.381 \pm 16.705$ & $-54.940 \pm 23.944$ & $122.307 \pm 23.076$ & $-14.046 \pm 0.895$ & $-74.060 \pm 12.541$ \\
ACE2-Eupatin & $-161.364 \pm 16.258$ & $-147.513 \pm 23.864$ & $255.502 \pm 28.956$ & $-18.165 \pm 0.737$ & $-71.540 \pm 24.104$ \\
MAPK3-Licochalcone & $-91.817 \pm 16.409$ & $-169.094 \pm 20.751$ & $206.876 \pm 28.292$ & $-14.699 \pm 1.102$ & $-68.734 \pm 17.678$ \\
$\quad$ B & & & & & $-138.106 \pm 12.919$ \\
TP53-Glycyrol & $-193.772 \pm 13.218$ & $-75.282 \pm 14.264$ & $149.156 \pm 15.680$ & $-18.208 \pm 1.034$ & $-75.794 \pm 14.521$ \\
PIK3CA-Epicatechin & $-141.128 \pm 11.715$ & $-68.751 \pm 8.263$ & $150.805 \pm 13.631$ & $-16.720 \pm 0.724$ & $-66.252 \pm 16.431$ \\
\hline JAK2-Aloe emodin & $-118.689 \pm 14.170$ & $-72.671 \pm 13.641$ & $140.475 \pm 14.320$ & $-15.367 \pm 0.987$ & \\
\hline
\end{tabular}

MAPK3-Licochalcone B, and JAK2-Aloe emodin complex, with free binding energy of $-75.794,-74.06,-71.54$, -68.734 and $-66.252 \mathrm{~kJ} / \mathrm{mol}$, respectively. To get a deeper understanding of the contribution of the binding pocket residues to the complex, the binding free energy decomposition per residue was employed (Fig. 13). The free energy decomposition analysis plots showed that Leu27, Cys 145 and Met165 were energetically favorable for the binding stability of 3CL-Hydroxygenkwanin complex, with the binding energy values of $-4.259,-6.819$ and $-3.485 \mathrm{~kJ} / \mathrm{mol}$, respectively. In contrast, Asp269, Trp271, His378, Glu402, Phe512 and Tyr515 were the major residues that interacted with ACE2-Eupatin and contributed to the total binding energy, with negative energy values of $-2.675,-5.872$, $-8.187,-7.114,-4.088$ and $-6.448 \mathrm{~kJ} / \mathrm{mol}$, respectively. However, Arg273, Glu375 and Arg514 showed positive energy values of $17.886,24.693$ and $8.233 \mathrm{~kJ} / \mathrm{mol}$, indicating no favorable binding to eupatin. All other complexes also exhibited a number of favorable and unfavorable residue energy contributions as described above.

\section{Discussion}

In this study, we systematically analyzed the chemical composition and therapeutic mechanism of XBCQD in the treatment of COVID-19 through network pharmacology, molecular docking and MD simulation. XBCQD is a classical prescription in TCM, which is composed of 4 Chinese medicines. Since Gypsum Fibrosum is a kind of sulfate mineral $\left(\mathrm{CaSO}_{4} \cdot 2 \mathrm{H}_{2} \mathrm{O}\right)$, many studies have excluded it in the experiments [61]. Considering Gypsum Fibrosum has a strong heat-clearing ability, and is used frequently in prescriptions for the treatment of COVID-19, Gypsum Fibrosum should also be included in the range of the study [62]. 


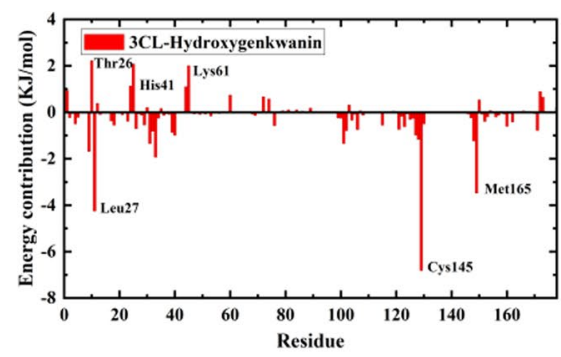

a

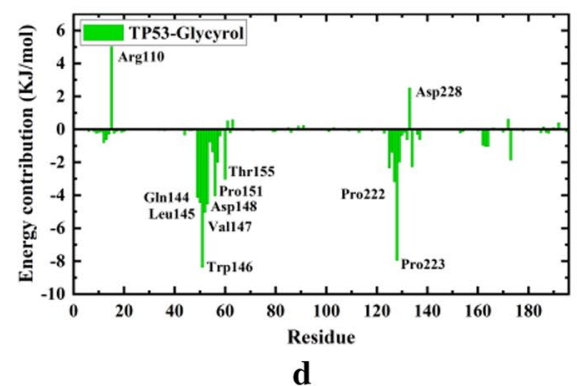

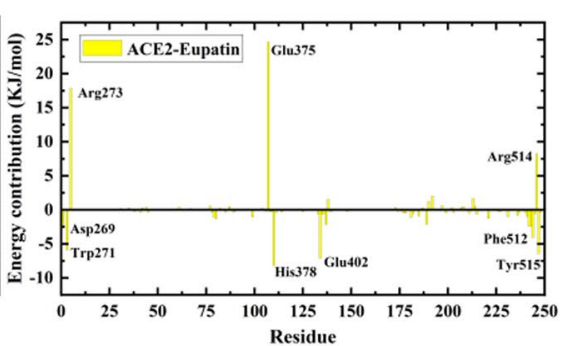

b

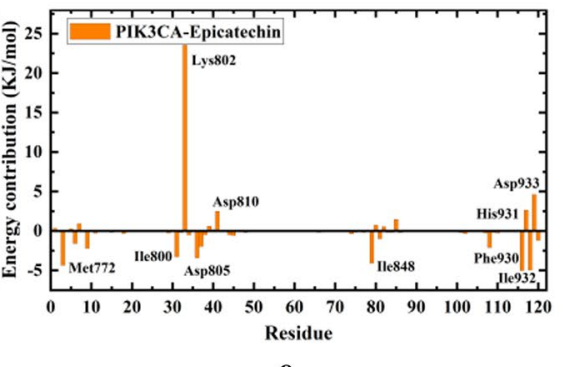

e

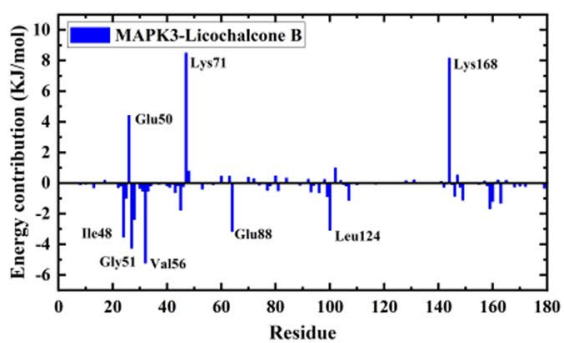

c

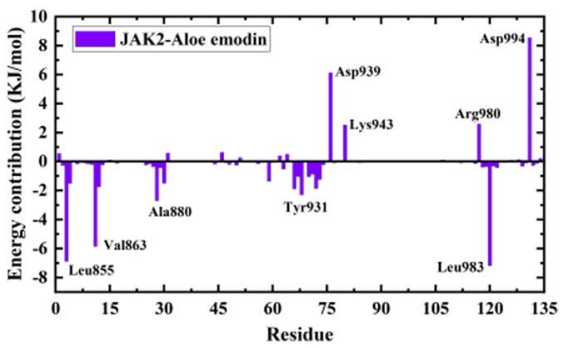

$\mathbf{f}$

Fig. 13 Per residue MM-PBSA energy contribution of complex systems in the $100 \mathrm{~ns}$ period of MD simulations

Based on the theory of TCM, effective compounds were identified from the ingredients of XBCQD using network pharmacology methods, and intricate networks were constructed for XBCQD to reveal the complex interactions, properties, and meridian tropisms among 4 kinds of Chinese medicines. Analyzing the meridian tropism network of XBCQD from a systemic perspective, 3 of the 4 Chinese medicines have a specific affinity to the lung, and the lung was the largest organ system degree node. These indicated that XBCQD mainly act on the lung system. According to research, SARS-CoV-2 mainly infects the host cells by targeting the highly expressed ACE2 receptor in the lung, causing lung tissue damage and respiratory tract insufficiency [63]. Our research provided meridian tropisms theory of TCM support for XBCQD in the treatment of COVID-19.

The 67 targets in the PPI network were scrutinized and 34 targets were extracted as hub targets, which usually have significant influence and play a more critical role in the network. RELA is a subunit of the transcription factor NF- $\mathrm{kB}$ and the hub target with the highest degree value, which indicated that NF- $\mathrm{KB}$ signaling pathway may be a therapeutic approach for XBCQD in the treatment of COVID-19. The nuclear factor NF- $\mathrm{KB}$ regulates inflammation, immunity, and cell survival, and is considered to be a typical proinflammatory signaling pathway [64]. Inhibiting the activation of NF- $\kappa \mathrm{B}$ can restore systemic hypotension, diminish intravascular coagulation, reduce tissue neutrophil influx, and prevent multiple organ injury [65]. Also, NF- $\mathrm{BB}$ induces the expression of inflammation and carcinogenesis proteins, such as IL2, TNF, and CXCL1 can cause inflammatory response and promote the survival and proliferation of lung tumor cells [66]. MAPK is involved in many cell physiological and pathological processes, such as cell growth, cell proliferation, cell differentiation and cell survival [67]. The phosphorylation of MAPK3/1 also enhances the co-transactivation of RELA. In addition, COVID-19 is closely related to cytokine storm [68]. Studies have shown that IL-6 is more relevant to the evaluation of COVID-19 patients and ICU patients, and their interleukin levels (IL-1 $\beta$, IL-2, IL-4, IL-6, IL-7, IL-8, IL-9, IL-10, IL-13, IL-17), interferon gamma, fibroblast growth factor and TNF are high [69]. Moreover, the serum levels of IL6, IL8, IL10 and TNF in severe patients are higher than those in mild patients, indicating that the severity of COVID-19 is associated with cytokine storm [70]. Therefore, the treatment based on the control of inflammatory factors may be beneficial to infected patients.

In order to further clarify the mechanism of XBCQD in the treatment of COVID-19, GO and KEGG enrichment was performed. The results of GO enrichment showed that the hub targets were mainly involved in the apoptotic, cytokine regulation and lipopolysaccharide response. KEGG enrichment analysis showed that the main pathway involves in viral infection and inflammatory response related signaling pathways. IL-17 is a family of multifunctional cytokines, which plays an important role in the development of inflammatory autoimmune diseases. It can upregulate proinflammatory factors via the activation of NF- $\mathrm{\kappa B}$ and MAPK [71]. In fact, the development of COVID-19 is closely associated with inflammation, apoptosis, and immune regulation. SARS-CoV-2 acts on the innate immune system, releasing chemokines and cytokines, which eventually lead to dyspnea and respiratory failure [72]. In the development of 
COVID-19, systemic inflammatory response and immune system disorders will not only affect cardiovascular system, but also damage lung or other parts of the body [73]. Recent studies have demonstrated that SARS-CoV-2 coding protein open reading frame 8 (ORF8) acted as a contributing factor to cytokine storm during COVID-19 infection. ORF8 could activate IL-17 signaling pathway and promote the expression of proinflammatory factors [74]. Therefore, targeting IL-17 can be used immunologically as a strategy to prevent acute respiratory distress syndrome in COVID-19.

Our current results showed that the effective compounds in XBCQD could regulate the inflammatory process and reduce lung tissue damage to exert its therapeutic effects on COVID-19. Furthermore, 10 core compounds were confirmed to have good drug-likeness and ADMET properties, which provided a basis on the drug-like capacity of a theoretical pharmaceutical. As a flavonoid, epicatechin has a variety of pharmacological activities, including antioxidant, anti-inflammatory and antitumor [75]. Studies have shown that epicatechin can reduce excessive inflammation and inhibits the activation of pro-inflammatory pathways by inhibiting the expression level of TNF- $\alpha$ and IL-6, and reduce acute lung injury [76]. Aloe-emodin is a natural anthraquinone in many Chinese herbal medicines, and has antioxidant and antibacterial activities [77]. Previous studies have shown that aloe-emodin can induce caspase-dependent apoptosis and autophagy. It also reduced the activity of nonsmall cell lung in a dose-dependent manner [78]. Moreover, aloe-emodin exerted anti-inflammatory effects by reducing the production of pro-inflammatory factors such as IL-6 and IL $1 \beta$ [79]. Diosmetin exhibits good anti-inflammatory effect. It has been reported that diosmetin inhibits IL-4 and LPS signaling pathways, and is effective in inhibiting Th2associated mast cells and in suppressing the expression of inflammatory cytokines [80]. Licochalcone B can induce cell cycle arrest, apoptosis, and defective autophagy, which inhibits growth and induces apoptosis of human non-smallcell lung cancer cells by dual targeting of EGFR and MET [81]. Glycyrol is an important anti-inflammatory and antivirus coumarin derivative. Glycyrol can decrease the mRNA of the IL- $1 \beta$ and IL- 6 in a dose-dependent manner, and inhibits I- $\kappa \mathrm{B} \alpha$ phosphorylation to prevent NF- $\mathrm{\kappa B}$ activation in RAW264.7 macrophages [82]. Eupatin has significantly anti-inflammatory effect. It can reduce the LPS-induced protein expression and phosphorylation of p65 and inducible nitric oxide synthase as well as downstream products IL 6 [83]. The stable molecular docking model exhibited effective combination between core compounds and hub targets, further verifying the internal relationship between XBCQD and COVID-19. MD simulations further analyzed the stability of the receptor-ligand complexes. The RMSD analysis showed that the six simulated systems basically reached the equilibrium after $100 \mathrm{~ns}$ of MD simulations. The residual flexibility was evaluated by the RMSF, which suggested that the regions with large fluctuations occurred mainly in protein terminal residues and loop regions. The average values of $\mathrm{Rg}$ for six complexes indicated compact receptor structures, suggesting that the binding of the core compounds to the targets had little effect on the protein structure. The hydrogen bond interactions explained the high stability and good binding affinity between core compounds and targets. Moreover, we computed the per-residue decomposition of free energy using the MM-PBSA method. The identification of key residues is of great help to further design inhibitors of SARS-CoV-2 targets.

The current results gave strong theoretical support for XBCQD in the treatment of COVID-19. Nevertheless, it should be noted that there were some limitations due to the lack of verification analyses in our research. The core compounds, hub targets and related pathways predicted by the computational tools require further in vitro and in vivo studies to validate the molecular mechanism of XBCQD for the treatment of COVID-19.

\section{Conclusions}

In this study, the chemical composition and therapeutic mechanism of XBCQD in the treatment of COVID-19 were investigated by combining network pharmacology, molecular docking, and MD simulations. According to our analysis, XBCQD could treat COVID-19 by modulating and targeting a variety of cytokines, and exerted potential therapeutic effects through anti-inflammatory and antiviral. The core compounds of XBCQD exhibited high potential for binding to the active site of hub targets, and the ligand-receptor complexes gradually reached stability with the extension of molecular simulation time. This research comprehensively explained the multi-component, multi-target, and multipathway intervention mechanism of XBCQD in the treatment of COVID-19, which was expected to provide evidence and new insights for further pharmacological research.

Supplementary Information The online version contains supplementary material available at https://doi.org/10.1007/s11030-022-10415-7.

Acknowledgements These works were supported by the Natural Science Basic Research Plan in Shaanxi Province of China(No.2020JZ-45), Natural Science Foundation of Shaanxi Province (No.2021JQ-935), Scientific Research Program of Shaanxi Provincial Education Department (No.18JS109), Student's Platform for Innovation and Entrepreneurship Training Program of Northwest University (No.2021463), and Novel Coronavirus Pneumonia Emergency Research Project of Northwestern University.

Author Contributions LF, SF, TW and YL participated in the research design. LF, KZ, ZW and MW responsible for data acquisition and analyses. LF, XD, XA and XZ assisted with data collection and manuscript 
revision. LF, SF, TW and YL wrote the manuscript and approved the final version of the manuscript.

Data availability All the data can be obtained from the open-source platform provided in the article.

\section{Declarations}

Conflict of interest The authors declare that they have no known competing financial interests in this paper.

\section{References}

1. Kong Q, Wu Y, Gu Y, Lv Q, Qi F, Gong S et al (2020) Analysis of the molecular mechanism of Pudilan (PDL) treatment for COVID-19 by network pharmacology tools. Biomed Pharmacother 128:110316. https://doi.org/10.1016/j.biopha.2020.110316

2. Chellasamy G, Arumugasamy SK, Govindaraju S, Yun K (2020) Analytical insights of COVID-19 pandemic. Trends Analyt Chem 133:116072. https://doi.org/10.1016/j.trac.2020.116072

3. Roberts CM, Levi M, McKee M, Schilling R, Lim WS, Grocott MPW (2020) COVID-19: a complex multisystem disorder. Brit J Anaesth 125(3):238-242. https://doi.org/10.1016/j.bja.2020.06. 013

4. Zheng S, Baak JP, Li S, Xiao W, Ren H, Yang H et al (2020) Network pharmacology analysis of the therapeutic mechanisms of the traditional Chinese herbal formula Lian Hua Qing Wen in Corona virus disease 2019 (COVID-19), gives fundamental support to the clinical use of LHQW. Phytomedicine 79:153336. https://doi.org/ 10.1016/j.phymed.2020.153336

5. Chen N, Zhou M, Dong X, Qu J, Gong F, Han Y et al (2020) Epidemiological and clinical characteristics of 99 cases of 2019 novel coronavirus pneumonia in Wuhan, China: a descriptive study. Lancet 395(10223):507-513. https://doi.org/10.1016/ S0140-6736(20)30211-7

6. Smail SW, Saeed M, Twana A, Khudhur ZO, Younus DA, Rajab MF et al (2021) Inflammation, immunity and potential target therapy of SARS-COV-2: a total scale analysis review. Food Chem Toxicol 150:112087. https://doi.org/10.1016/j.fct.2021.112087

7. Li G, De Clercq E (2020) Therapeutic options for the 2019 novel coronavirus (2019-nCoV). Nat Rev Drug Discov 19(3):149-150. https://doi.org/10.1038/d41573-020-00016-0

8. Chen J, Wang YK, Gao Y, Hu LS, Yang JW, Wang JR et al (2020) Protection against COVID-19 injury by qingfei paidu decoction via anti-viral, anti-inflammatory activity and metabolic programming. Biomed Pharmacother 129:110281. https://doi.org/10. 1016/j.biopha.2020.110281

9. Mao Z, Wang H (2016) Effects of Xuanbai Chengqi decoction on lung compliance for patients with exogenous pulmonary acute respiratory distress syndrome. Drug Des Devel Ther 10:793-798. https://doi.org/10.2147/DDDT.S93165

10. Liu MA, Zhong XG, Li YH, Zheng FJ, Wu RH, Sun Y et al (2014) Xuan Bai Cheng Qi formula as an adjuvant treatment of acute exacerbation of chronic obstructive pulmonary disease of the syndrome type phlegm-heat obstructing the lungs: a multicenter, randomized, double-blind, placebo-controlled clinical trial. Bmc Complem Altern M 14:239. https://doi.org/10.1186/ 1472-6882-14-239

11. Ren X, Shao XX, Li XX, Jia XH, Song T, Zhou WY et al (2020) Identifying potential treatments of COVID-19 from traditional chinese medicine (TCM) by using a data-driven approach. J Ethnopharmacol 258:112932. https://doi.org/10.1016/j.jep.2020. 112932
12. Zhu Y, Xia W, Liu W, Xu C, Gu N (2017) Gualoupi (Pericarpium Trichosanthis) injection in combination with convention therapy for the treatment of angina pectoris: a Meta- analysis. J Tradit Chin Med 37(1):1-11. https://doi.org/10.1016/s0254-6272(17) 30020-1

13. Li Y, Chu F, Li P, Johnson N, Li T, Wang Y et al (2021) Potential effect of Maxing Shigan decoction against coronavirus disease 2019 (COVID-19) revealed by network pharmacology and experimental verification. J Ethnopharmacol 271:113854. https://doi. org/10.1016/j.jep.2021.113854

14. Mu S, Zhang J, Du S, Zhu M, Wei W, Xiang J et al (2021) Gut microbiota modulation and anti-inflammatory properties of Xuanbai Chengqi decoction in septic rats. J Ethnopharmacol 267:113534. https://doi.org/10.1016/j.jep.2020.113534

15. Zhao Z, Li Y, Zhou L, Zhou X, Xie B, Zhang W et al (2021) Prevention and treatment of COVID-19 using traditional chinese medicine: a review. Phytomedicine 85:153308. https://doi.org/10. 1016/j.phymed.2020.153308

16. Ang L, Lee HW, Choi JY, Zhang JH, Lee MS (2020) Herbal medicine and pattern identification for treating COVID-19: a rapid review of guidelines. Integr Med Res. https://doi.org/10.1016/j. imr.2020.100407

17. Luo TT, Lu Y, Yan SK, Xiao X, Rong XL, Guo J (2020) Network pharmacology in research of chinese medicine formula: methodology, application and prospective. Chin J Integr Med 26(1):72-80. https://doi.org/10.1007/s11655-019-3064-0

18. Pagadala NS, Syed K, Tuszynski J (2017) Software for molecular docking: a review. Biophys Rev 9(2):91-102. https://doi.org/10. 1007/s12551-016-0247-1

19. Kondori J, Zendehboudi S, Hossain ME (2017) A review on simulation of methane production from gas hydrate reservoirs: molecular dynamics prospective. J Petrol Sci Eng 159:754-772. https://doi.org/10.1016/j.petrol.2017.09.073

20. Ru J, Li P, Wang J, Zhou W, Li B, Huang C et al (2014) TCMSP: a database of systems pharmacology for drug discovery from herbal medicines. J Cheminformatics 6:13. https://doi.org/10. 1186/1758-2946-6-13

21. Liu Z, Guo F, Wang Y, Li C, Zhang X, Li H et al (2016) BATMAN-TCM: a bioinformatics analysis tool for molecular mechanism of traditional Chinese medicine. Sci Rep 6:21146. https:// doi.org/10.1038/srep21146

22. Xu X, Zhang W, Huang C, Li Y, Yu H, Wang Y et al (2012) A novel chemometric method for the prediction of human oral bioavailability. Int J Mol Sci 13(6):6964-6982. https://doi.org/10. 3390/ijms 13066964

23. Walters JR (2002) New advances in the molecular and cellular biology of the small intestine. Curr Opin Gastroenterol 18(2):161167. https://doi.org/10.1097/00001574-200203000-00002

24. Wang JF, Zhou H, Han LY, Chen X, Chen YZ, Cao ZW (2005) Traditional Chinese medicine information database. Clin Pharmacol Ther 78(1):92-93. https://doi.org/10.1016/j.clpt.2005.03.010

25. Xu HY, Zhang YQ, Liu ZM, Chen T, Lv CY, Tang SH et al (2019) ETCM: an encyclopaedia of traditional Chinese medicine. Nucleic Acids Res 47(D1):D976-D982. https://doi.org/10.1093/nar/ gky987

26. Daina A, Michielin O, Zoete V (2019) SwissTargetPrediction: updated data and new features for efficient prediction of protein targets of small molecules. Nucleic Acids Res 47(W1):W357W364. https://doi.org/10.1093/nar/gkz382

27. Wang X, Shen Y, Wang S, Li S, Zhang W, Liu X et al (2017) PharmMapper 2017 update: a web server for potential drug target identification with a comprehensive target pharmacophore database. Nucleic Acids Res 45(W1):W356-W360. https://doi.org/10. 1093/nar/gkx374

28. Yao ZJ, Dong J, Che YJ, Zhu MF, Wen M, Wang NN et al (2016) TargetNet: a web service for predicting potential 
drug-target interaction profiling via multi-target SAR models. J Comput Aid Mol Des 30(5):413-424. https://doi.org/10.1007/ s10822-016-9915-2

29. Liangzi F, Qinfang Z, Jun H (2021) Research on saponin active compounds of Tuchao Baibiandouren for the treatment of type-2 diabetes based on UHPLC-Q-Exactive Orbitrap MS and network pharmacology. Digit Chin Med 4(1):19-31. https://doi. org/10.1016/j.dcmed.2021.03.003

30. Stelzer G, Rosen N, Plaschkes I, Zimmerman S, Twik M, Fishilevich $S$ et al (2016) The genecards suite: from gene data mining to disease genome sequence analyses. Curr Protoc Bioinform. https://doi.org/10.1002/cpbi.5

31. UniProt C (2021) UniProt: the universal protein knowledgebase in 2021. Nucleic Acids Res 49(D1):D480-D489. https://doi.org/ 10.1093/nar/gkaa1100

32. Szklarczyk D, Gable AL, Nastou KC, Lyon D, Kirsch R, Pyysalo S et al (2021) The STRING database in 2021: customizable protein-protein networks, and functional characterization of user-uploaded gene/measurement sets. Nucleic Acids Res 49(18):10800. https://doi.org/10.1093/nar/gkaa1074

33. Alshehri B, Vijayakumar R, Senthilkumar S, Ismail A, Abdelhadi A, Choudhary RK et al (2022) Molecular target prediction and docking of anti-thrombosis compounds and its activation on tissue-plasminogen activator to treat stroke. J King Saud Univ Sci. https://doi.org/10.1016/j.jksus.2021.101732

34. Xiong G, Wu Z, Yi J, Fu L, Yang Z, Hsieh C et al (2021) ADMETlab 2.0: an integrated online platform for accurate and comprehensive predictions of ADMET properties. Nucleic Acids Res 49(W1):W5-W14. https://doi.org/10.1093/nar/gkab2 55

35. Swargiary G, Mani S (2022) ER and PGR targeting ability of phytocompounds derived from Centella asiatica and Andrographis paniculata: an in-silico approach. J Herb Med. https://doi. org/10.1016/j.hermed.2022.100541

36. Kim S, Thiessen PA, Bolton EE, Chen J, Fu G, Gindulyte A et al (2016) PubChem substance and compound databases. Nucleic Acids Res 44(D1):D1202-D1213. https://doi.org/10.1093/nar/ gkv951

37. Berman HM, Westbrook J, Feng Z, Gilliland G, Bhat TN, Weissig $\mathrm{H}$ et al (2000) The protein data bank. Nucleic Acids Res 28(1):235-242. https://doi.org/10.1093/nar/28.1.235

38. Shan Y, Dong J, Pan X, Zhang L, Zhang J, Dong Y et al (2015) Expanding the structural diversity of Bcr-Abl inhibitors: dibenzoylpiperazin incorporated with $1 \mathrm{H}$-indazol-3-amine. Eur J Med Chem 104:139-147. https://doi.org/10.1016/j.ejmech.2015.09. 034

39. Liu R, Su C, Xu Y, Shang K, Sun K, Li C et al (2020) Identifying potential active components of walnut leaf that action diabetes mellitus through integration of UHPLC-Q-Orbitrap HRMS and network pharmacology analysis. J Ethnopharmacol 253:112659. https://doi.org/10.1016/j.jep.2020.112659

40. Wang L, Li H, Shen X, Zeng J, Yue L, Lin J et al (2020) Elucidation of the molecular mechanism of Sanguisorba Officinalis L. against leukopenia based on network pharmacology. Biomed Pharmacother 132:110934. https://doi.org/10.1016/j.biopha.2020. 110934

41. Abraham MJ, Murtola T, Schulz R, Páll S, Smith JC, Hess B et al (2015) GROMACS: High performance molecular simulations through multi-level parallelism from laptops to supercomputers. SoftwareX 1-2:19-25. https://doi.org/10.1016/j.softx.2015.06. 001

42. Stroet M, Caron B, Visscher KM, Geerke DP, Malde AK, Mark AE (2018) Automated topology builder version 3.0: prediction of solvation free enthalpies in water and hexane. J Chem Theory Comput 14(11):5834-5845. https://doi.org/10.1021/acs.jctc. $8 \mathrm{~b} 00768$
43. Tom D, Darrin Y, Lee P (1993) Particle mesh Ewald: an $\mathrm{N} \cdot \log (\mathrm{N})$ method for Ewald sums in large systems. J Chem Phys 98(12):10089. https://doi.org/10.1063/1.464397

44. Kumari R, Kumar R, Lynn A (2014) g_mmpbsa-a GROMACS tool for high-throughput MM-PBSA calculations. J Chem Inf Model 54(7):1951-1962. https://doi.org/10.1021/ci500020m

45. Kumari P, Vijayan R, Gourinath S (2021) Structural analysis of EhPSP in complex with 3-phosphoglyceric acid from Entamoeba histolytica reveals a basis for its lack of phosphoglycerate mutase activity. Int J Biol Macromol 178:1-10. https://doi.org/10.1016/j. ijbiomac.2021.02.153

46. Choi SB, Ko BS, Park SK, Jang JS, Park S (2006) Insulin sensitizing and alpha-glucoamylase inhibitory action of sennosides, rheins and rhaponticin in Rhei Rhizoma. Life Sci 78(9):934-942. https://doi.org/10.1016/j.lfs.2005.05.101

47. Wang Y, Yang X, Xia P, Ma X, Yang R, Hu J et al (2019) Research progress on chemical composition and pharmacological effects of Rhei Radix et Rhizoma and predictive analysis on quality markers. Chin Tradit Herb Drugs 50(19):4821-4837

48. Wan GZ, Ma XH, Jin L, Chen J (2021) alpha-glucosidase immobilization on magnetic core-shell metal-organic frameworks for inhibitor screening from traditional Chinese medicines. Colloid Surf B 205:111847. https://doi.org/10.1016/j.colsurfb.2021. 111847

49. Ngoc TM, Minh PT, Hung TM, Thuong PT, Lee I, Min BS et al (2008) Lipoxygenase inhibitory constituents from rhubarb. Arch Pharm Res 31(5):598-605. https://doi.org/10.1007/ s12272-001-1199-0

50. He XY, Wu LJ, Wang WX, Xie PJ, Chen YH, Wang F (2020) Amygdalin - A pharmacological and toxicological review. J Ethnopharmacol 254:112717. https://doi.org/10.1016/j.jep.2020. 112717

51. Tsai YW, Lu CH, Chang RC, Hsu YP, Ho LT, Shih KC (2021) Palmitoleic acid ameliorates palmitic acid-induced proinflammation in J774A.1 macrophages via TLR4-dependent and TNF-alphaindependent signallings. Prostag Leukotr Ess 169:102270. https:// doi.org/10.1016/j.plefa.2021.102270

52. He H, Guo Q (2019) Research progress on chemical composition and pharmacological effects of Trichosanthis Fructus and predictive analysis on quality marker. Chin Tradit Herb Drugs 50(19):4808-4820

53. An HM, Huang DR, Yang H, Liu XG, Du J, Li Y et al (2020) Comprehensive chemical profiling of Jia-Wei-Qi-Fu-Yin and its network pharmacology-based analysis on Alzheimer's disease. J Pharmaceut Biomed 189:113467. https://doi.org/10.1016/j.jpba. 2020.113467

54. Li T, Zeng H, Zeng Y, Zhang X, Ren Y, Gao Y et al (2021) Characterization of the bioactive compounds with efficacy against gout in Guizhi Shaoyao Zhimu Decoction by UHPLC-Q-Orbitrap HRMS combined with network pharmacological analysis. Arab J Chem 14(6):103185. https://doi.org/10.1016/j.arabjc.2021.103185

55. Schwandner R, Yamaguchi K, Cao Z (2000) Requirement of tumor necrosis factor receptor-associated factor (TRAF)6 in interleukin 17 signal transduction. J Exp Med 191(7):1233-1239. https://doi. org/10.1084/jem.191.7.1233

56. Kunnumakkara AB, Rana V, Parama D, Banik K, Girisa S, Henamayee $\mathrm{S}$ et al (2021) COVID-19, cytokines, inflammation, and spices: How are they related? Life Sci 284:119201. https://doi. org/10.1016/j.lfs.2021.119201

57. Zhang H, Penninger JM, Li Y, Zhong N, Slutsky AS (2020) Angiotensin-converting enzyme 2 (ACE2) as a SARS-CoV-2 receptor: molecular mechanisms and potential therapeutic target. Intens Care Med 46(4):586-590. https://doi.org/10.1007/ s00134-020-05985-9

58. Tahir Ul Qamar M, Alqahtani SM, Alamri MA, Chen LL (2020) Structural basis of SARS-CoV-2 3CL(pro) and anti-COVID-19 
drug discovery from medicinal plants. J Pharm Anal 10(4):313319. https://doi.org/10.1016/j.jpha.2020.03.009

59. Zhang L, Lin D, Sun X, Curth U, Drosten C, Sauerhering L et al (2020) Crystal structure of SARS-CoV-2 main protease provides a basis for design of improved alpha-ketoamide inhibitors. Science 368(6489):409-412. https://doi.org/10.1126/science.abb3405

60. Hoffmann M, Kleine-Weber H, Schroeder S, Kruger N, Herrler T, Erichsen S et al (2020) SARS-CoV-2 cell entry depends on ACE2 and TMPRSS2 and is blocked by a clinically proven protease inhibitor. Cell 181(2):271-280. https://doi.org/10.1016/j. cell.2020.02.052

61. Yao Y, He Z, Liu X, He Y, Lei Y, Zhang S et al (2020) Potential material basis of kangbingdu granules for treatment of coronavirus disease 2019 (COVID-19) based on network pharmacology and molecular docking technology. Chin Tradit Herb Drugs 51(6):1386-1396

62. Sun X, Jiang J, Wang Y, Liu S (2020) Exploring the potential therapeutic effect of traditional Chinese medicine on coronavirus disease 2019 (COVID-19) through a combination of data mining and network pharmacology analysis. Eur J Integr Med 40:101242. https://doi.org/10.1016/j.eujim.2020.101242

63. Mehrabadi ME, Hemmati R, Tashakor A, Homaei A, Yousefzadeh M, Hemati K et al (2021) Induced dysregulation of ACE2 by SARS-CoV-2 plays a key role in COVID-19 severity. Biomed Pharmacother 137:111363. https://doi.org/10.1016/j.biopha.2021. 111363

64. Lawrence $T$ (2009) The nuclear factor NF-kappaB pathway in inflammation. Csh Perspect Biol 1(6):a1651. https://doi.org/10. 1101/cshperspect.a001651

65. Liu SF, Malik AB (2006) NF-kappa B activation as a pathological mechanism of septic shock and inflammation. Am J Physiol Lung C 290(4):L622-L645. https://doi.org/10.1152/ajplung.00477.2005

66. He T, Shen HY, Wang SM, Wang YF, He ZW, Zhu LT et al (2020) MicroRNA-3613-5p promotes lung adenocarcinoma cell proliferation through a RELA and AKT/MAPK positive feedback loop. Mol Ther-Nucl Acids 22:572-583. https://doi.org/10.1016/j.omtn. 2020.09.024

67. Xiong H, Dong Z, Lou G, Gan Q, Wang J, Huang Q (2020) Analysis of the mechanism of Shufeng Jiedu capsule prevention and treatment for COVID-19 by network pharmacology tools. Eur J Integr Med 40:101241. https://doi.org/10.1016/j.eujim.2020. 101241

68. Huang C, Wang Y, Li X, Ren L, Zhao J, Hu Y et al (2020) Clinical features of patients infected with 2019 novel coronavirus in Wuhan China. Lancet 395(10223):497-506. https://doi.org/10. 1016/S0140-6736(20)30183-5

69. Gao Y, Li T, Han M, Li X, Wu D, Xu Y et al (2020) Diagnostic utility of clinical laboratory data determinations for patients with the severe COVID-19. J Med Virol 92(7):791-796. https://doi.org/ 10.1002/jmv. 25770

70. Qin C, Zhou L, Hu Z, Zhang S, Yang S, Tao Y et al (2020) Dysregulation of immune response in patients with coronavirus 2019 (COVID-19) in Wuhan. China. Clin Infect Dis 71(15):762-768. https://doi.org/10.1093/cid/ciaa248

71. Zhu S, Qian Y (2012) IL-17/IL-17 receptor system in autoimmune disease: mechanisms and therapeutic potential. Clin Sci 122(11):487-511. https://doi.org/10.1042/CS20110496

72. Zhang C, Wu Z, Li JW, Zhao H, Wang GQ (2020) Cytokine release syndrome in severe COVID-19: interleukin-6 receptor antagonist tocilizumab may be the key to reduce mortality. Int J Antimicrob Ag 55(5):105954. https://doi.org/10.1016/j.ijantimicag.2020.105954

73. Zheng YY, Ma YT, Zhang JY, Xie X (2020) COVID-19 and the cardiovascular system. Nat Rev Cardiol 17(5):259-260. https:// doi.org/10.1038/s41569-020-0360-5

74. Lin X, Fu B, Yin S, Li Z, Liu H, Zhang H et al (2021) ORF8 contributes to cytokine storm during SARS-CoV-2 infection by activating IL-17 pathway. iScience 24(4):102293. https://doi.org/ 10.1016/j.isci.2021.102293

75. Prakash M, Basavaraj BV, Murthy KNC (2019) Biological functions of epicatechin: plant cell to human cell health. J Funct Foods 52:14-24. https://doi.org/10.1016/j.jff.2018.10.021

76. Xing J, Yu Z, Zhang X, Li W, Gao D, Wang J et al (2019) Epicatechin alleviates inflammation in lipopolysaccharide-induced acute lung injury in mice by inhibiting the p38 MAPK signaling pathway. Int Immunopharmacol 66:146-153. https://doi.org/10. 1016/j.intimp.2018.11.016

77. Gao Y, Zhang XY, Li XM, Qi RJ, Han YX, Kang Y et al (2021) Aloe-emodin, a naturally occurring anthraquinone, is a highly potent mast cell stabilizer through activating mitochondrial calcium uniporter. Biochem Pharmacol 186:114476. https://doi.org/ 10.1016/j.bcp.2021.114476

78. Shen F, Ge C, Yuan P (2020) Aloe-emodin induces autophagy and apoptotic cell death in non-small cell lung cancer cells via Akt/mTOR and MAPK signaling. Eur J Pharmacol 886:173550. https://doi.org/10.1016/j.ejphar.2020.173550

79. Hu B, Zhang H, Meng X, Wang F, Wang P (2014) Aloe-emodin from rhubarb (Rheum rhabarbarum) inhibits lipopolysaccharideinduced inflammatory responses in RAW264.7 macrophages. J Ethnopharmacol 153(3):846-853. https://doi.org/10.1016/j.jep. 2014.03.059

80. Lee DH, Park JK, Choi J, Jang H, Seol JW (2020) Anti-inflammatory effects of natural flavonoid diosmetin in IL-4 and LPSinduced macrophage activation and atopic dermatitis model. Int Immunopharmacol 89:107046. https://doi.org/10.1016/j.intimp. 2020.107046

81. Oh HN, Lee MH, Kim E, Yoon G, Chae JI, Jung-Hyun S (2019) Licochalcone B inhibits growth and induces apoptosis of human non-small-cell lung cancer cells by dual targeting of EGFR and MET. Phytomedicine 63:153014. https://doi.org/10.1016/j. phymed.2019.153014

82. Shin EM, Zhou HY, Guo LY, Kim JA, Lee SH, Merfort I et al (2008) Anti-inflammatory effects of glycyrol isolated from Glycyrrhiza uralensis in LPS-stimulated RAW264.7 macrophages. Int Immunopharmacol 8(11):1524-1532. https://doi.org/10.1016/j. intimp.2008.06.008

83. Chou CH, Hsu KC, Lin TE, Yang CR (2020) Anti-inflammatory and tau phosphorylation-inhibitory effects of eupatin. Molecules 25(23):5652. https://doi.org/10.3390/molecules25235652

Publisher's Note Springer Nature remains neutral with regard to jurisdictional claims in published maps and institutional affiliations. 